Supporting Information for:

\title{
Adsorption of organic compounds by biomass chars: direct role of aromatic condensation (ring cluster size) revealed by experimental and theoretical studies
}

Jingjing Yang ${ }^{1}$, Joseph J. Pignatello ${ }^{2 *}$, Kun Yang ${ }^{3}$, Wenhao Wu ${ }^{3}$, Guining Lu ${ }^{1,4}$, Lijuan Zhang ${ }^{5}$, Chen Yang ${ }^{1,4 *}$, Zhi Dang ${ }^{1,4}$

Submitted to

Environmental Science \& Technology

${ }^{1}$ School of Environment and Energy, South China University of Technology, Guangzhou 510006, China

${ }^{2}$ Department of Environmental Sciences, The Connecticut Agricultural Experiment Station, 123 Huntington St., New Haven, Connecticut, 06511, United States

${ }^{3}$ Department of Environmental Science, Zhejiang University, Hangzhou 310058,

China

4 Key Laboratory of Pollution Control and Ecosystem Restoration in Industry Clusters, Ministry of Education, Guangdong, Guangzhou 510006, China

${ }^{5}$ School of Chemistry and Chemical Engineering, South China University of Technology, Guangzhou 510006, China

*Corresponding author:

cyanggz@scut.edu.cn (Chen Yang); joseph.pignatello@ct.gov (Joseph J. Pignatello) 
Text S1 Preparation and characterization of chars

$4 \quad$ Table S1. List and selected physicochemical properties of the test compounds

5 Table S2. Select physical-chemical properties of chars.

6 Table S3. Dubinin Ashtahkov model parameters for test compounds on chars.

7 Table S4. Results of linear regressions between $\boldsymbol{E}_{\mathbf{D A}}$ and fraction of bridgehead carbon: $\boldsymbol{E}_{\mathbf{D A}}=m_{\mathrm{DA}} \boldsymbol{\chi}_{\mathbf{b}}+$ const $_{\mathrm{DA}}$

9 Table S5. The average site energy $\boldsymbol{E}_{\mathbf{D A}, \text { mean }}\left(\mathrm{kJ} \mathrm{mol}^{-1}\right)$ of chars for 22 organic compounds.

Table S6. Resutls of linear regression between sorption capacity $\left(\boldsymbol{Q}^{0}\right)$ and $\chi_{\mathbf{b}}$

11 Table S7. Computational binding energies $\left(\boldsymbol{E}_{\mathbf{b d}}\right)$ and minimum distances for the sheet-compound 12 configurations shown in Fig. S13

Table S8. Results of linear regression between $\boldsymbol{E}_{\mathbf{b d}}$ and fraction of bridgehead aromatic carbon: $\boldsymbol{E}_{\mathbf{b d}}=$

$$
m_{\mathrm{bd}} \chi_{\mathbf{b}}+\text { const }_{\mathrm{bd}}
$$

Table S9. Static polarizability components and polarizability of polybenzeneoid hydrocarbons

Table S10. Computational binding energies $\left(\boldsymbol{E}_{\mathbf{b d}}\right)$ of phenol adsorption between dual parallel PBH sheets (Ba400, D) at different intersheet distances $(\mathrm{Ba} 400, \mathbf{D})$

Table S11. Binding energies $\left(\boldsymbol{E}_{\mathbf{b d}}\right)$ of phenol adsorption between dual parallel PBH sheets $(\mathrm{Ba} 400, \mathbf{D})$ at an inter-sheet distance of $0.68 \mathrm{~nm}$

Table S12. Binding energies $\left(\boldsymbol{E}_{\mathbf{b d}}\right)$ of two parallel-planar molecules adsorbed in a bilayer between parallel $\mathrm{PBH}$ sheets placed $1 \mathrm{~nm}$ apart.

Figure S1. Solid state ${ }^{13} \mathrm{C}$ DP/MAS NMR spectra of chars.

Figure S2. Correlation between the total aromatic carbon content $\left(F_{\text {ar }}\right)$ and atomic ratio of $\mathrm{H} / \mathrm{C}$ for chars.

Figure S3. Preliminary kinetic experiments for two compounds with different solubilities at two different concentrations on $\mathrm{Ba} 550, \mathrm{Ba} 400$ and $\mathrm{Ba} 250$ which represents chars produced at high HTT, medium HTT and low HTT, respectively. 
Figure S4. Adsorption isotherms of anilines on chars fit to the Dubinin-Ashtakhov model.

Figure S5. Adsorption isotherms of phenols on chars fit to the Dubinin-Ashtakhov model.

Figure S6. Adsorption isotherms of nitrobenzenes and PAHs on chars fit to the Dubinin-Ashtakhov model.

Figure S7. Correlations between the fraction of aromatic carbon $\left(\boldsymbol{F}_{\mathbf{a r}}\right)$ and $\boldsymbol{E}_{\mathbf{D A}}$ values. Solid lines connect Ba200 and Ba250 and dotted lines are the extraploted line to the $\boldsymbol{F}_{\text {ar }}$ corresponding to fresh bamboo (0.12, red arrows).

Figure S8. Correlations between fraction of bridgehead aromatic carbon and $\boldsymbol{E}_{\mathbf{D A}}$ values. Solid lines are linear regression fits of $\mathrm{Ba} 300, \mathrm{Ba} 400, \mathrm{Ba} 550$ and $\mathrm{Ba} 700$. Dash lines are extrapolated lines from the solid lines.

Figure S9. Correlations between fraction of bridgehead carbon and $\boldsymbol{E}_{\mathbf{D A}}$, mean values listed in Table S5.

Figure S10. Correlations between sorption $\boldsymbol{E}_{\mathbf{D A}}$ values and fraction of bridgehead aromatic carbon $\left(\chi_{\mathbf{b}}\right)$ on wood chars. Data is obtained from the literatures ${ }^{1,2}$.

Figure S11 Correlations between sorption capacity $\left(\boldsymbol{Q}^{\mathbf{0}}, \mathrm{mg}^{-1}\right)$ and fraction of bridgehead aromatic carbon $\left(\chi_{\mathbf{b}}\right)$.

Figure S12. Correlations between the slope $m_{\mathrm{DA}}$ of the relationship $\boldsymbol{E}_{\mathbf{D A}}=m_{\mathrm{DA}} \chi_{\mathbf{b}}+$ const $_{\mathrm{DA}}$ (from Table S4) and, (a) the sum of Hammett $\sigma_{\text {meta }}$ (b) the sum of Hammett $\sigma_{\text {para }}$ or (c) the molecular weight, for 22 compounds.

Figure S13. Simulation results for the optimized complexes. (dark gray: C; white: H; blue: N; red: O). The PBH sheets correspond, left to right, to the chars: B200, Ba250, Ba300, Ba400, Ba550, and Ba700 (structures A-F, Fig. 4a).

Figure S14. Correlations between static polarizability of PBH $\mathbf{A}-\mathbf{H}$ (Fig. 4a) and $\boldsymbol{E}_{\mathbf{b d}}$ for adsorption of four compounds on the single-sheet $\mathrm{PBH}$.

Figure S15. Correlations between micropore volume and $\boldsymbol{E}_{\mathbf{D A}}$ values. Solid lines are linear fits.

Figure S16. Correlations between $\mathrm{CO}_{2}-\mathrm{SSA}$ and $\boldsymbol{E}_{\mathbf{D A}}$ values. Solid lines are linear fits.

Figure S17. Correlations between fraction of bridgehead aromatic carbon and surface area and micropore volume. Short dot lines are linear fits. 


\section{Text S1 Preparation and characterization of chars}

Chars were prepared by placing a covered ceramic crucible tightly compacted to capacity with bamboo chips in a muffle furnace held at $200,250,300,400,550$, or $700{ }^{\circ} \mathrm{C}$ for $6 \mathrm{~h}$. No effort was made to control furnace headspace gas composition, so some leakage of air into the crucible during pyrolysis was likely. This is realistic since biochars and natural chars are seldom formed under strictly anoxic conditions. Chars were demineralized with $1 \mathrm{M} \mathrm{HCl}$ solution for $12 \mathrm{~h}$, rinsed repeatedly with ultrapure water (Milli-Q, Millipore), and dried at $80{ }^{\circ} \mathrm{C}$ before storage in sealed glass containers. Experiments commenced several months after production. Elemental analysis (Table S2) was measured using a Flash EA 1112 CHN elemental analyzer (Thermo Finningan). Micropore size distribution (Fig. 1b) and specific surface area (SSA) (Table S2) were calculated by DFT and Monte Carlo Methods from the $\mathrm{CO}_{2}$ adsorption isotherm (Autosorb IQ-MP Viton, Anton-Paar, USA) at $273 \mathrm{~K}$ following outgassing at $453 \mathrm{~K}$. $\mathrm{N}_{2}$ B.E.T. surface area of chars (Table S2) were calculated based $\mathrm{N}_{2}$ isotherms at $77 \mathrm{~K}$ by Brunauer-Emmett-Teller (B.E.T.) theory.

\section{Text S2 Sorption}

Batch adsorption experiments were conducted in 40-ml (naphthalene), 100-ml (phenanthrene), 250-ml (pyrene), or 8-ml (all other compounds) flame-sealed glass ampules. Solid-to-solution ratios were adjusted to achieve at least $20 \%$ uptake of added compound. Stock solutions of test compounds were prepared in purified water containing $0.01 \mathrm{M} \mathrm{CaCl}_{2}$, except for PAHs, which were prepared in methanol and then diluted to different initial concentrations with $0.01 \mathrm{M} \mathrm{CaCl}_{2}$. The volume ratio of methanol to water in the final solution was kept below $0.5 \%$ to avoid cosolvent effects. Water used in this study was purified by a Milli-Q system $(18.2 \mathrm{M} \Omega \bullet \mathrm{cm})$. To maintain the phenols in their neutral form, the $\mathrm{pH}$ of the mixtures was adjusted with dilute $\mathrm{NaOH}$ or $\mathrm{HCl}$ : to $\mathrm{pH} 4.0$ for 2-chlorophenol, 2,4-dichlorophenol, 2-nitrophenol, 3-nitrophenol, and 4-nitrophenol; or to $\mathrm{pH} 8.0$ for aniline and 4-methylphenol. For other compounds, the final solution $\mathrm{pH}$ was maintained at 6.5-7.5. The suspensions were placed in a rotating mixer at 150 rotations per minute for 5 days. Preliminary kinetic experiments showed 5 days was 
82 sufficient to reach a plateau in the liquid-phase concentration (Figure S3). After the 5-day period, the 83 solid and solution phases were separated by centrifugation (3500 revolutions per min, $20 \mathrm{~min}$ ). 84 Liquid-phase concentrations of PAHs were quantified using Agilent 1200 high-performance liquid 85 chromatography with a reverse-phase $\mathrm{C}_{18}$ column and a fluorescence detector. Concentrations of other 86 chemicals were determined by UV-visible spectrophotometry (Shimadzu, UV-2450, Tokyo, Japan) at 87 their maximum absorption wavelengths listed in Table S1. The uncertainties in the measurements were 88 below 4\%. The sorbed concentration was calculated by the difference between the amount added and the 89 amount remaining in solution.

90

91

92

93

94

95

96

97

98

99

100

101

102

103

104 
Table S1. List and selected physicochemical properties of the test compounds (sources and purities were given previously. ${ }^{3}$ )

\begin{tabular}{|c|c|c|c|c|c|c|c|}
\hline \multirow[t]{2}{*}{ Compounds } & \multirow[t]{2}{*}{ MW a } & \multirow[t]{2}{*}{$\lambda_{\max } \mathrm{b}$} & \multirow[t]{2}{*}{$\mathrm{p} K_{\mathrm{a}}^{\mathrm{c}}$} & \multirow[t]{2}{*}{$T_{\text {melting }} \mathrm{d}$} & \multirow[t]{2}{*}{$\Delta \mathrm{H}^{\mathrm{e}}$} & \multirow[t]{2}{*}{$C_{\mathrm{w}}^{\mathrm{sat}}(s)^{\mathrm{f}}$} & $\begin{array}{c}\text { Sub-cooled } \\
\text { water } \\
\text { solubility }\end{array}$ \\
\hline & & & & & & & $C_{\mathrm{W}}^{\mathrm{sg}} \angle \square$ \\
\hline Aniline & 93.13 & 230 & 4.6 & -6.2 & 1 & 34160 & / \\
\hline 2-chloroaniline & 128.58 & 232 & 2.66 & -1.9 & / & 5700 & l \\
\hline 2-nitroaniline & 138.13 & 223 & -0.29 & 71.2 & 16110 & 1260 & 3013 \\
\hline 3-nitroaniline & 138.13 & 225 & 2.5 & 114 & 23680 & 900 & 8089 \\
\hline 4-nitroaniline & 138.13 & 380 & 1 & 147 & 21090 & 600 & 7095 \\
\hline Phenol & 94.11 & 269 & 9.9 & 40.9 & 11290 & 80190 & 100987 \\
\hline 2-chlorophenol & 128.56 & 273 & 8.44 & 9.8 & l & 28500 & l \\
\hline 4-chlorophenol & 128.56 & 225 & 9.38 & 42.7 & 14070 & 26300 & 36149 \\
\hline 4-methylphenol & 108.13 & 276 & 10.14 & 35.8 & 11890 & 23000 & 27198 \\
\hline 2-nitrophenol & 139.11 & 277 & 7.17 & 44.8 & 17440 & 2100 & 3254 \\
\hline 3-nitrophenol & 139.11 & 273 & 8.28 & 96.8 & 19200 & 14000 & 62942 \\
\hline 4-nitrophenol & 139.11 & 317 & 7.15 & 113.8 & 18250 & 16000 & 86661 \\
\hline 2,4-dichlorophenol & 163 & 245 & 7.9 & 44.8 & 20090 & 4600 & 7619 \\
\hline Nitrobenzene & 123.11 & 269 & / & 5.7 & I & 1936 & / \\
\hline 4-nitrotoluene & 137.14 & 284 & I & 51.6 & 16810 & 340 & 592 \\
\hline 4-chloronitrobenzene & 157.56 & 279 & / & 83.5 & 20780 & 224.8 & 889 \\
\hline 1,2-dinitrobenzene & 168.11 & 255 & l & 116.9 & 22680 & 133 & 1148 \\
\hline 1,3-dinitrobenzene & 168.11 & 242 & / & 89.7 & 17370 & 574.9 & 2005 \\
\hline 1,4-dinitrobenzene & 168.11 & 266 & l & 173.5 & 28130 & 69 & 3000 \\
\hline Naphthalene & 128.18 & $\begin{array}{c}\text { Excitation:218; } \\
\text { Emission:324 }\end{array}$ & / & 78.2 & 19120 & 31.7 & 102 \\
\hline Phenanthrene & 178.23 & $\begin{array}{c}\text { Excitation:250; } \\
\text { Emission:366 }\end{array}$ & l & 99.2 & 16470 & 1.29 & 4.85 \\
\hline Pyrene & 202.26 & $\begin{array}{c}\text { Excitation:238; } \\
\text { Emission:373 }\end{array}$ & I & 151.2 & 17110 & 0.135 & 1.05 \\
\hline
\end{tabular}

a MW: molecular weight $\left(\mathrm{g} \mathrm{mol}^{-1}\right) ;{ }^{\mathbf{b}} \lambda_{\max }:$ maximum absorption wavelength (nm); ${ }^{\mathbf{c}} K_{\mathrm{a}}:$ dissociated constant; ${ }^{\mathbf{d}} T_{\text {melting: }}$ : melting points of compounds, ${ }^{\circ} \mathrm{C} ;{ }^{\text {e }}$ Molar enthalpy of fusion $\left(\mathrm{J} \mathrm{mol}^{-1}\right)$, data from reference $1{ }^{4} ;{ }_{\mathrm{W}}^{\mathrm{f}} C_{\mathrm{W}}^{\text {sat }}(s)$ : observed water solubility at $25{ }^{\circ} \mathrm{C}\left(\mathrm{mg} \mathrm{L}^{-1}\right)$;

g Subcool water solubility $\left(\mathrm{mg} \mathrm{L}^{-1}\right)$ for compounds in the crystalline state at $298 \mathrm{~K}$ was calculated by the equations:

$\Delta_{\text {fus }} G=\frac{\Delta \bar{H}_{\mathrm{A}}^{\text {fus }} \cdot\left(T_{\mathrm{mp}}-T\right)}{T_{\mathrm{mp}}}$ and $C_{\mathrm{w}}^{\text {sat }}(L)=C_{\mathrm{w}}^{\text {sat }}(s) \cdot \exp \left(\Delta_{\text {fus }} G / R T\right) 1,4$ 
Table S2. Select physical-chemical properties of chars. ${ }^{a}$

\begin{tabular}{|c|c|c|c|c|c|c|c|c|c|c|c|c|c|}
\hline Char & $\begin{array}{c}\mathrm{C} \\
(\%)\end{array}$ & $\begin{array}{c}\mathrm{H} \\
(\%)\end{array}$ & $\begin{array}{c}\mathrm{O} \\
(\%)\end{array}$ & $\begin{array}{c}\mathrm{N} \\
(\%)\end{array}$ & $\begin{array}{l}\text { Ash } \\
(\%)\end{array}$ & $\mathrm{H} / \mathrm{C}$ & $\begin{array}{c}\mathrm{N}_{2} \text { B.E.T. } \\
\left(\mathrm{m}^{2} \mathrm{~g}^{-1}\right)\end{array}$ & $\begin{array}{c}\mathrm{CO}_{2} \text { specific } \\
\text { surface area, } \\
\mathrm{CO}_{2}-\mathrm{SSA} \\
\left(\mathrm{m}^{2} \mathrm{~g}^{-1}\right)\end{array}$ & $\begin{array}{l}\mathrm{CO}_{2} \text { pore } \\
\text { volume, } \\
V_{\text {micropore }} \\
\left(\mathrm{cm}^{3} \mathrm{~g}^{-1}\right)\end{array}$ & $\begin{array}{c}\text { Total aromatic } \\
\text { C content, } F_{a r} \\
(\%)\end{array}$ & $F_{\mathrm{ar}, \mathrm{b}}(\%)$ & $\chi_{b}^{c}$ & $\begin{array}{c}\text { Ave number } \\
\text { of C per ring } \\
\text { cluster }\end{array}$ \\
\hline $\mathrm{Ba} 200$ & 52.6 & 5.67 & 40.9 & 0.070 & 0.72 & 1.29 & 4.56 & 91.4 & 0.034 & 38.0 & 8.2 & 0.22 & 11 \\
\hline $\mathrm{Ba} 250$ & 60.6 & 4.46 & 33.0 & 0.421 & 1.49 & 0.883 & 4.82 & 160 & 0.053 & 56.0 & 16.6 & 0.30 & 15 \\
\hline $\mathrm{Ba} 300$ & 71.2 & 3.90 & 24.0 & 0.260 & 0.65 & 0.710 & 5.39 & 307 & 0.090 & 71.0 & 28.0 & 0.39 & 20 \\
\hline $\mathrm{Ba} 400$ & 72.3 & 2.72 & 21.4 & 0.679 & 2.94 & 0.451 & 7.36 & 402 & 0.112 & 81.6 & 36.3 & 0.45 & 22 \\
\hline Ba550 & 79.4 & 2.18 & 14.0 & 0.963 & 3.49 & 0.329 & 448 & 545 & 0.162 & 89.9 & 47.8 & 0.53 & 28 \\
\hline $\mathrm{Ba} 700$ & 87.2 & 1.43 & 7.44 & 0.525 & 3.38 & 0.197 & 511 & 636 & 0.187 & 85.8 & 59.1 & 0.69 & 63 \\
\hline
\end{tabular}

a: The $\mathrm{C}, \mathrm{H}$, and $\mathrm{N}$ contents were measured using a Flash EA $1112 \mathrm{CHN}$ elemental analyzer (Thermo Finningan). Ash contents were determined by heating the biochar at $850{ }^{\circ} \mathrm{C}$ for $1 \mathrm{~h}$ in a muffle furnace. The oxygen content was calculated by mass difference. Nitrogen B.E.T. specific surface area (Table S2) was calculated by the Brunauer-Emmett-Teller (BET) theory for $\mathrm{N}_{2}$ adsorption isotherm at $0.05 \leq p / p_{0} \leq 0.3$. The $\mathrm{N}_{2}$ B.E.T. specific surface area is complicated by the kinetic inaccessibility of some of the pores at $77 \mathrm{~K}^{5} 6$. 
Table S3. Dubinin-Ashtahkov model parameters for select test compounds on chars a

\begin{tabular}{|c|c|c|c|c|c|c|}
\hline Compounds & Char & $\begin{array}{c}\log Q^{0} \\
\left(Q^{0}, \mathrm{mg} \mathrm{g}^{-1}\right)\end{array}$ & $\begin{array}{c}\boldsymbol{E}_{\mathbf{D A}} \\
\left(\mathrm{kJ} \mathrm{mol}^{-1}\right)\end{array}$ & $b$ & $r^{2}$ & $\mathrm{~N}$ \\
\hline \multirow[t]{6}{*}{ Aniline } & $\mathrm{Ba} 200$ & $2.445 \pm 0.151$ & $7.04 \pm 1.46$ & $0.76 \pm 0.11$ & 0.998 & 15 \\
\hline & $\mathrm{Ba} 250$ & $2.518 \pm 0.045$ & $11.4 \pm 0.59$ & $0.87 \pm 0.06$ & 0.999 & 15 \\
\hline & $\mathrm{Ba} 300$ & $2.480 \pm 0.032$ & $14.7 \pm 0.53$ & $0.87 \pm 0.04$ & 0.999 & 15 \\
\hline & $\mathrm{Ba} 400$ & $2.173 \pm 0.016$ & $19.0 \pm 0.45$ & $1.37 \pm 0.09$ & 0.993 & 21 \\
\hline & $\mathrm{Ba} 550$ & $2.204 \pm 0.010$ & $24.7 \pm 0.75$ & $1.44 \pm 0.08$ & 0.990 & 23 \\
\hline & Ba700 & $2.218 \pm 0.003$ & $31.6 \pm 0.25$ & $1.98 \pm 0.04$ & 0.998 & 28 \\
\hline \multirow[t]{6}{*}{ Phenol } & $\mathrm{Ba} 200$ & $2.593 \pm 0.030$ & $6.66 \pm 0.24$ & $0.97 \pm 0.03$ & 0.998 & 24 \\
\hline & $\mathrm{Ba} 250$ & $2.612 \pm 0.017$ & $9.50 \pm 0.20$ & $0.97 \pm 0.02$ & 0.999 & 24 \\
\hline & $\mathrm{Ba} 300$ & $2.561 \pm 0.011$ & $13.7 \pm 0.17$ & $1.04 \pm 0.02$ & 0.999 & 24 \\
\hline & $\mathrm{Ba} 400$ & $2.220 \pm 0.015$ & $17.9 \pm 0.23$ & $1.75 \pm 0.10$ & 0.990 & 24 \\
\hline & $\mathrm{Ba} 550$ & $2.201 \pm 0.007$ & $26.8 \pm 0.20$ & $1.79 \pm 0.06$ & 0.996 & 24 \\
\hline & Ba700 & $2.262 \pm 0.083$ & $33.3 \pm 0.26$ & $2.26 \pm 0.08$ & 0.995 & 21 \\
\hline \multirow[t]{6}{*}{ 4-nitrophenol } & $\mathrm{Ba} 200$ & $2.994 \pm 0.043$ & $8.27 \pm 0.33$ & $1.07 \pm 0.03$ & 0.999 & 24 \\
\hline & $\mathrm{Ba} 250$ & $2.954 \pm 0.030$ & $11.2 \pm 0.36$ & $1.01 \pm 0.03$ & 0.999 & 24 \\
\hline & $\mathrm{Ba} 300$ & $2.648 \pm 0.024$ & $18.2 \pm 0.42$ & $1.19 \pm 0.03$ & 0.999 & 24 \\
\hline & $\mathrm{Ba} 400$ & $2.344 \pm 0.015$ & $25.0 \pm 0.25$ & $1.71 \pm 0.06$ & 0.998 & 24 \\
\hline & $\mathrm{Ba} 550$ & $2.343 \pm 0.005$ & $38.1 \pm 0.16$ & $1.68 \pm 0.03$ & 0.999 & 24 \\
\hline & Ba700 & $2.249 \pm 0.079$ & $51.4 \pm 1.20$ & $2.11 \pm 0.08$ & 0.995 & 26 \\
\hline \multirow[t]{6}{*}{ Nitrobenzene } & $\mathrm{Ba} 200$ & $1.928 \pm 0.014$ & $6.44 \pm 0.10$ & $0.92 \pm 0.02$ & 1.000 & 23 \\
\hline & $\mathrm{Ba} 250$ & $2.262 \pm 0.013$ & $8.90 \pm 0.11$ & $0.92 \pm 0.02$ & 1.000 & 24 \\
\hline & $\mathrm{Ba} 300$ & $2.454 \pm 0.026$ & $12.9 \pm 0.32$ & $0.90 \pm 0.05$ & 0.998 & 20 \\
\hline & $\mathrm{Ba} 400$ & $2.208 \pm 0.012$ & $17.2 \pm 0.19$ & $1.29 \pm 0.05$ & 0.998 & 22 \\
\hline & $\mathrm{Ba} 550$ & $2.302 \pm 0.010$ & $28.0 \pm 0.75$ & $1.33 \pm 0.07$ & 0.992 & 24 \\
\hline & $\mathrm{Ba} 700^{\mathrm{a}}$ & $2.266 \pm 0.003$ & $34.4 \pm 0.44$ & $2.24 \pm 0.06$ & 0.995 & 19 \\
\hline \multirow[t]{6}{*}{ Naphthalene } & $\mathrm{Ba} 200$ & $2.072 \pm 0.218$ & $5.27 \pm 1.20$ & $0.91 \pm 0.12$ & 0.995 & 20 \\
\hline & $\mathrm{Ba} 250$ & $1.642 \pm 0.072$ & $10.6 \pm 0.73$ & $1.17 \pm 0.10$ & 0.993 & 22 \\
\hline & $\mathrm{Ba} 300$ & $1.431 \pm 0.036$ & $14.2 \pm 0.29$ & $1.96 \pm 0.14$ & 0.994 & 18 \\
\hline & $\mathrm{Ba} 400$ & $1.575 \pm 0.037$ & $16.8 \pm 0.46$ & $1.45 \pm 0.11$ & 0.992 & 21 \\
\hline & $\mathrm{Ba} 550$ & $2.040 \pm 0.015$ & $27.1 \pm 0.35$ & $1.65 \pm 0.09$ & 0.993 & 19 \\
\hline & Ba700 & $2.079 \pm 0.010$ & $32.0 \pm 0.19$ & $3.23 \pm 0.12$ & 0.994 & 21 \\
\hline
\end{tabular}

${ }^{a}$ Estimated parameter values and their standard errors were determined by commercial software (Origin 8.0) using nonlinear regression 
Table S4. Results of linear regressions between $E_{\mathrm{DA}}$ and fraction of bridgehead carbon: $\boldsymbol{E}_{\mathbf{D A}}=m_{\mathrm{DA}} \boldsymbol{\chi}_{\mathbf{b}}+$ const $_{\mathrm{DA}}$

\begin{tabular}{cccc}
\hline Compounds & $m_{\mathrm{DA}}$ & const $_{\mathrm{DA}}$ & $r^{2}$ \\
\hline aniline & $55.6 \pm 5.51$ & $-6.14 \pm 2.90$ & 0.97 \\
2-chloroaniline & $63.1 \pm 11.7$ & $-8.83 \pm 6.17$ & 0.9 \\
2-nitroaniline & $50.8 \pm 8.81$ & $-2.13 \pm 4.64$ & 0.91 \\
3-nitroaniline & $51.3 \pm 12.2$ & $1.87 \pm 6.41$ & 0.85 \\
4-nitroaniline & $66.6 \pm 10.0$ & $-5.37 \pm 7.27$ & 0.94 \\
phenol & $66.3 \pm 10.5$ & $-11.2 \pm 5.53$ & 0.93 \\
2-chlorophenol & $88.4 \pm 10.1$ & $-19.8 \pm 5.3$ & 0.96 \\
4-chlorophenol & $88.0 \pm 17.4$ & $-19.6 \pm 9.17$ & 0.89 \\
4-methylphenol & $80.7 \pm 20.4$ & $-17.2 \pm 10.8$ & 0.83 \\
2,4-dichlorophenol & $89.0 \pm 14.9$ & $-21.6 \pm 7.86$ & 0.92 \\
2-nitrophenol & $69.2 \pm 13.6$ & $-11.7 \pm 7.17$ & 0.89 \\
3-nitrophenol & $108 \pm 11.1$ & $-25.5 \pm 5.86$ & 0.97 \\
4-nitrophenol & $112 \pm 11.8$ & $-24.4 \pm 6.24$ & 0.97 \\
nitrobenzene & $73.3 \pm 13.8$ & $-14.6 \pm 7.28$ & 0.9 \\
4-nitrotoluene & $69.6 \pm 3.27$ & $-12.1 \pm 1.72$ & 0.99 \\
4-chloronitrobenzne & $63.7 \pm 13.6$ & $-8.25 \pm 7.15$ & 0.77 \\
1,2-dinitrobenzene & $55.7 \pm 9.66$ & $-5.99 \pm 5.09$ & 0.91 \\
1,3-dinitrobenzene & $81.7 \pm 5.98$ & $-15.9 \pm 3.15$ & 0.98 \\
1,4-dinitrobenzene & $65.1 \pm 13.4$ & $-6.30 \pm 7.07$ & 0.84 \\
naphthalene & $62.0 \pm 13.4$ & $-9.39 \pm 7.09$ & \\
phenanthrene & $59.7 \pm 6.25$ & $-8.01 \pm 3.30$ & $-5.95 \pm 6.96$ \\
pyrene & $53.5 \pm 13.2$ & & 0.97 \\
\hline & & & \\
\hline
\end{tabular}


Table S5. The average site energy $\boldsymbol{E}_{\mathbf{D A}, \text { mean }}\left(\mathrm{kJ} \mathrm{mol}^{-1}\right)$ of chars for 22 organic compounds.

\begin{tabular}{|c|c|c|c|c|c|c|}
\hline Compounds & $\mathrm{Ba} 200$ & $\mathrm{Ba} 250$ & $\mathrm{Ba} 300$ & $\mathrm{Ba} 400$ & $\mathrm{Ba} 550$ & Ba700 \\
\hline Phenol & 4.84 & 6.14 & 7.34 & 7.77 & 9.98 & 33.4 \\
\hline 2-chlorophenol & 10.5 & 5.74 & 7.63 & 8.59 & 9.77 & 13.3 \\
\hline 4-chlorophenol & 13.4 & 6.66 & 7.64 & 8.42 & 9.02 & 13.5 \\
\hline 4-methylphenol & 4.5 & 6.63 & 8.27 & 8.46 & 10.6 & 13.4 \\
\hline 2,4-dichlorophenol & 6.00 & 7.78 & 8.37 & 8.73 & 11.4 & 11.5 \\
\hline 2-nitrophenol & 5.32 & 6.66 & 8.27 & 8.33 & 11.2 & 12.4 \\
\hline 3-nitrophenol & 8.23 & 9.24 & 11.6 & 11.9 & 14.1 & 16.0 \\
\hline 4-nitrophenol & 9.32 & 10.2 & 14.2 & 13.6 & 15.6 & 16.7 \\
\hline Aniline & 5.76 & 6.80 & 9.12 & 7.59 & 8.98 & 12.4 \\
\hline 2-chloroaniline & 4.83 & 6.12 & 9.04 & 6.73 & 8.07 & 10.7 \\
\hline 2-nitroaniline & 6.64 & 8.42 & 9.68 & 10.2 & 10.5 & 13.8 \\
\hline 3-nitroaniline & 10.7 & 12.3 & 14.6 & 12.3 & 12.6 & 15.9 \\
\hline 4-nitroaniline & 10.4 & 12.0 & 13.3 & 14.1 & 16.4 & 19.5 \\
\hline Nitrobenzene & 4.26 & 5.74 & 6.85 & 7.96 & 10.3 & 13 \\
\hline 4-nitrotoluene & 5.92 & 6.56 & 8.68 & 9.64 & 10.3 & 12.0 \\
\hline 4-chloronitrobenzene & 8.96 & 9.41 & 10.3 & 12.4 & 13.9 & 14.9 \\
\hline 1,2-dinitrobenzene & 10.2 & 10.8 & 12.8 & 11.6 & 12.7 & 15.3 \\
\hline 1,3-dinitrobenzene & 7.30 & 8.58 & 9.74 & 10.6 & 11.6 & 13.6 \\
\hline 1,4-dinitrobenzene & 13.9 & 14.9 & 16.7 & 16.3 & 18.1 & 19.0 \\
\hline Naphthalene & 8.49 & 7.74 & 8.58 & 9.29 & 11.2 & 13.7 \\
\hline Phenanthrene & 6.23 & 6.69 & 7.61 & 8.14 & 9.86 & 12.4 \\
\hline Pyrene & 9.76 & 10.7 & 10.1 & 9.86 & 11.0 & 11.6 \\
\hline
\end{tabular}

$\boldsymbol{E}_{\mathbf{D A} \text {,mean }}$ were calculated from equations those are cited from ref ${ }^{7}$ :

$\boldsymbol{E}_{\mathrm{DA}, \text { mean }}=\frac{\int_{\varepsilon_{\mathrm{h}}}^{\varepsilon_{\mathrm{1}}} \frac{\left(\varepsilon / \boldsymbol{E}_{\mathrm{DA}}\right)^{b}}{10^{\left[\left(\varepsilon / E_{\mathrm{DA}}\right)^{b}\right]}} d_{\varepsilon}}{\int_{\varepsilon \mathrm{h}}^{\varepsilon_{1}} \frac{\varepsilon^{b-1}}{\boldsymbol{E}_{\mathrm{DA}}^{b} \boldsymbol{g} 0^{\left[\left(\varepsilon / \boldsymbol{E}_{\mathrm{DA}}\right)^{b}\right]}} d_{\varepsilon}}$

$\varepsilon=-R T \ln \frac{C_{e}}{C_{\mathrm{W}}^{\text {sat }}(L)}$

where $C_{\mathrm{e}}(\mathrm{mg} / \mathrm{L})$ is the equilibrium aqueous concentration; $C_{\mathrm{W}}^{\mathrm{sat}}(L)\left(\mathrm{mg} \mathrm{L}^{-1}\right)$ is the liquid or sub-cooled liquid water solubility at $298 \mathrm{~K}, R=8.314 \times 10^{-3} \mathrm{~kJ} /(\mathrm{mol} \mathrm{K}) ; T=298 \mathrm{~K} . \varepsilon_{1}$ and $\varepsilon_{\mathrm{h}}$ are $\varepsilon$ values at the lowest concentration and the highest concentration in the experimental concentration range, respectively. 
Table S6. Resutls of linear regression between sorption capacity $\left(\boldsymbol{Q}^{0}\right)$ and $\chi_{\mathbf{b}}$

\begin{tabular}{cccccc}
\hline Compounds & $r^{2}$ & $p$ value & Compounds & $r^{2}$ & $p$ value \\
\hline Aniline & 0.57 & 0.08 & 4-nitrophenol & 0.78 & 0.02 \\
2-chloroaniline & 0.41 & 0.17 & 2,4-dichlorophenol & 0.674 & 0.05 \\
2-nitroaniline & 0.04 & 0.17 & Nitrobenzene & 0.12 & 0.51 \\
3-nitroaniline & 0.08 & 0.58 & 4-nitrotoluene & 0.55 & 0.09 \\
4-nitroaniline & 0.14 & 0.47 & 4-chloronitrobenzene & 0.88 & $<0.01$ \\
Phenol & 0.67 & 0.05 & 1,2-dinitrobenzene & 0.12 & 0.50 \\
2-chlorophenol & 0.73 & 0.03 & 1,3-dinitrobenzene & 0.54 & 0.10 \\
4-chlorophenol & 0.71 & 0.04 & 1,4-dinitrobenzene & 0.76 & 0.02 \\
4-methylphenol & 0.11 & 0.53 & Naphthalene & 0.09 & 0.56 \\
2-nitrophenol & 0.11 & 0.52 & Phenanthrene & 0.16 & 0.43 \\
3-nitrophenol & 0.35 & 0.22 & Pyrene & 0.22 & 0.34 \\
\hline
\end{tabular}

All linear regression and significance test were obtained by a commercial software program (SPSS 19.0).

Table S7. Computational binding energies $\left(\boldsymbol{E}_{\mathbf{b d}}\right)$ and minimum distances for the sheet-compound configurations shown in Fig. S13

\begin{tabular}{|c|c|c|c|c|c|}
\hline & $\begin{array}{r}\text { Sheet } \\
(\mathrm{Ha})\end{array}$ & $\begin{array}{l}\text { Compound } \\
\text { (Ha) }\end{array}$ & $\begin{array}{c}\text { Sheet-Compound } \\
\text { (Ha) }\end{array}$ & $\begin{array}{c}\boldsymbol{E}_{\mathbf{b d}} \\
\left(\mathrm{kJ} \mathrm{mol}^{-1}\right)\end{array}$ & $\begin{array}{c}\text { Distance }^{\mathrm{b}} \\
(\AA)\end{array}$ \\
\hline \multicolumn{6}{|c|}{ Phenol } \\
\hline $\mathrm{Ba} 200^{\mathrm{a}}$ & -385.5381 & -307.2240 & -692.7678 & -14.7 & 3.380 \\
\hline $\mathrm{Ba} 250$ & -539.0425 & -307.2240 & -846.2741 & -19.7 & 3.400 \\
\hline $\mathrm{Ba} 300$ & -615.2223 & -307.2240 & -922.4561 & -25.2 & 3.262 \\
\hline $\mathrm{Ba} 400$ & -844.9077 & -307.2240 & -1152.1438 & -31.2 & 3.242 \\
\hline Ba550 & -1150.7556 & -307.2240 & -1457.9940 & -37.9 & 3.174 \\
\hline Ba700 & -2067.1767 & -307.2240 & -2374.4182 & -46.0 & 3.148 \\
\hline $\mathrm{C}_{66} \mathrm{H}_{20}$ & -2525.3754 & -307.2240 & -2832.6170 & -46.1 & 3.093 \\
\hline $\mathrm{C}_{90} \mathrm{H}_{24}$ & -3441.7623 & -307.2240 & -3749.0042 & -46.9 & 3.033 \\
\hline \multicolumn{6}{|c|}{ Aniline } \\
\hline $\mathrm{Ba} 200$ & -385.5381 & -287.3591 & -672.9046 & -19.5 & 3.311 \\
\hline $\mathrm{Ba} 250$ & -539.0425 & -287.3591 & -826.4123 & -28.0 & 3.236 \\
\hline $\mathrm{Ba} 300$ & -615.2223 & -287.3591 & -902.5936 & -29.8 & 3.210 \\
\hline $\mathrm{Ba} 400$ & -844.9077 & -287.3591 & -1132.2814 & -38.5 & 3.209 \\
\hline Ba550 & -1150.7556 & -287.3591 & -1438.1312 & -43.2 & 3.142 \\
\hline Ba700 & -2067.1767 & -287.3591 & -2354.5553 & -51.2 & 3.100 \\
\hline $\mathrm{C}_{66} \mathrm{H}_{20}$ & -2525.3754 & -287.3591 & -2812.7527 & -47.7 & 3.128 \\
\hline $\mathrm{C}_{90} \mathrm{H}_{24}$ & -3441.7623 & -287.3591 & -3729.1410 & -51.3 & 3.109 \\
\hline \multicolumn{6}{|c|}{ Nitrobenzene } \\
\hline $\mathrm{Ba} 200$ & -385.5381 & -436.4499 & -821.9991 & -29.3 & 3.310 \\
\hline & & & $\mathrm{S} 11$ & & \\
\hline
\end{tabular}




\begin{tabular}{|c|c|c|c|c|c|}
\hline $\mathrm{Ba} 250$ & -539.0425 & -436.4499 & -975.5041 & -30.8 & 3.267 \\
\hline Вa300 & -615.2223 & -436.4499 & -1051.6867 & -38.4 & 3.218 \\
\hline $\mathrm{Ba} 400$ & -844.9077 & -436.4499 & -1281.3731 & -40.9 & 3.255 \\
\hline $\mathrm{Ba} 550$ & -1150.7556 & -436.4499 & -1587.2232 & -46.6 & 3.239 \\
\hline $\mathrm{Ba} 700$ & -2067.1767 & -436.4499 & -2503.6469 & -53.5 & 3.154 \\
\hline $\mathrm{C}_{66} \mathrm{H}_{20}$ & -2525.3754 & -436.4499 & -2961.8456 & -53.3 & 3.103 \\
\hline $\mathrm{C}_{90} \mathrm{H}_{24}$ & -3441.7623 & -436.4499 & -3878.2323 & -52.8 & 3.081 \\
\hline \multicolumn{6}{|c|}{ Naphthalene } \\
\hline $\mathrm{Ba} 200$ & -385.5381 & -385.5379 & -771.0834 & -19.2 & 3.377 \\
\hline $\mathrm{Ba} 250$ & -539.0425 & -385.5379 & -924.5910 & -27.7 & 3.335 \\
\hline $\mathrm{Ba} 300$ & -615.2223 & -385.5379 & -1000.7734 & -35.7 & 3.266 \\
\hline $\mathrm{Ba} 400$ & -844.9077 & -385.5379 & -1230.4629 & -45.6 & 3.230 \\
\hline $\mathrm{Ba} 550$ & -1150.7556 & -385.5379 & -1536.3134 & -52.2 & 3.228 \\
\hline Ba700 & -2067.1767 & -385.5379 & -2452.7403 & -67.5 & 3.143 \\
\hline $\mathrm{C}_{66} \mathrm{H}_{20}$ & -2525.3754 & -385.5379 & -2910.9401 & -70.3 & 3.092 \\
\hline $\mathrm{C}_{90} \mathrm{H}_{24}$ & -3441.7623 & -385.5379 & -3827.3281 & -73.0 & 3.098 \\
\hline \multicolumn{6}{|c|}{ 4-Nitrophenol } \\
\hline $\mathrm{Ba} 200$ & -385.5381 & -511.6499 & -897.2000 & -31.3 & 3.249 \\
\hline $\mathrm{Ba} 250$ & -539.0425 & -511.6499 & -1050.7052 & -33.4 & 3.406 \\
\hline $\mathrm{Ba} 300$ & -615.2223 & -511.6499 & -1126.8858 & -37.4 & 3.226 \\
\hline $\mathrm{Ba} 400$ & -844.9077 & -511.6499 & -1356.5742 & -43.6 & 3.190 \\
\hline $\mathrm{Ba} 550$ & -1150.7556 & -511.6499 & -1662.4216 & -42.3 & 3.237 \\
\hline Ba700 & -2067.1767 & -511.6499 & -2578.8487 & -57.9 & 3.050 \\
\hline $\mathrm{C}_{66} \mathrm{H}_{20}$ & -2525.3754 & -511.6499 & -3037.0465 & -55.6 & 3.114 \\
\hline $\mathrm{C}_{90} \mathrm{H}_{24}$ & -3441.7623 & -511.6499 & -3953.4331 & -54.7 & 3.124 \\
\hline
\end{tabular}

${ }^{a} \mathrm{Ba} 200-\mathrm{Ba} 700$ and $\mathrm{C}_{66} \mathrm{H}_{20}$ and $\mathrm{C}_{90} \mathrm{H}_{24}$ correspond, respectively, to structures A-H in Fig.4a of main paper.

${ }^{\mathrm{b}}$ Distance from the center of the benzene ring of the compounds to the plane of the aromatic sheet.

Table S8. Results of linear regression between $E_{\mathrm{bd}}$ and mole fraction of bridgehead aromatic carbon: $\boldsymbol{E}_{\mathbf{b d}}=m_{\mathrm{bd}} \chi_{\mathbf{b}}+$ const $_{\mathrm{bd}}$

\begin{tabular}{cccc}
\hline Compounds & $m_{\mathrm{bd}}$ & const $_{\text {bd }}$ & $r^{2}$ \\
\hline Phenol & $69.3 \pm 2.88$ & $0.32 \pm 1.28$ & 0.99 \\
4-nitrophenol & $55.0 \pm 8.02$ & $18.0 \pm 3.56$ & 0.90 \\
Nitrobenzene & $54.9 \pm 3.89$ & $17.0 \pm 1.73$ & 0.98 \\
Aniline & $68.0 \pm 4.83$ & $6.70 \pm 2.14$ & 0.98 \\
Naphthalene & $104 \pm 1.90$ & $-2.21 \pm 0.84$ & 1.00 \\
\hline
\end{tabular}


Table S9. Static polarizability components and polarizability of polybenzeneoid hydrocarbons

\begin{tabular}{ccccc}
\hline Char & $\alpha_{\mathrm{xx}}(\mathrm{au})$ & $\alpha_{\mathrm{yy}}(\mathrm{au})$ & $\alpha_{\mathrm{zz}}(\mathrm{au})$ & $\alpha_{\mathrm{PBH}}\left(\AA^{3}\right)$ \\
\hline $\mathrm{Ba} 200$ & 176.94 & 128.35 & 66.60 & 18.37 \\
$\mathrm{Ba} 250$ & 307.07 & 173.14 & 87.62 & 28.05 \\
$\mathrm{Ba} 300$ & 388.77 & 273.29 & 108.54 & 38.06 \\
$\mathrm{Ba} 400$ & 499.64 & 448.10 & 139.66 & 53.71 \\
$\mathrm{Ba} 550$ & 591.67 & 542.60 & 155.54 & 63.71 \\
$\mathrm{Ba} 700$ & 1149.09 & 1148.42 & 249.59 & 125.81 \\
$\mathrm{C}_{66} \mathrm{H}_{20}$ & 1634.15 & 1400.71 & 295.92 & 164.52 \\
$\mathrm{C}_{90} \mathrm{H}_{24}$ & 2798.80 & 2031.57 & 388.43 & 257.78 \\
\hline
\end{tabular}

Ba200-Ba700 correspond, respectively, to structures A-H in Fig.4a of main paper. $\alpha_{\mathrm{PBH}}=\left(\alpha_{\mathrm{xx}}+\alpha_{\mathrm{yy}}+\right.$ $\left.\alpha_{\mathrm{zz}}\right) / 3$. au, atomic units.

Table S10. Computational binding energies $\left(\boldsymbol{E}_{\mathbf{b d}}\right)$ of phenol adsorption between dual parallel PBH sheets $(\mathrm{Ba} 400, \mathbf{D})$ at different intersheet distances $(\mathrm{Ba} 400, \mathbf{D})$

\begin{tabular}{ccccc}
\hline Distance $(\mathrm{nm})$ & Sheet $(\mathrm{Ha})$ & Phenol $(\mathrm{Ha})$ & Sheet-Phenol $(\mathrm{Ha})$ & $\boldsymbol{E}_{\mathbf{b d}}\left(\mathrm{kJ} \mathrm{mol}^{-1}\right)$ \\
\hline 0.45 & -1689.8218 & -307.2240 & -1996.7854 & 683 \\
0.50 & -1689.8181 & -307.2240 & -1996.9432 & 260 \\
0.55 & -1689.8210 & -307.2240 & -1997.0279 & 45.0 \\
0.58 & -1689.8197 & -307.2240 & -1997.0500 & -18.7 \\
0.60 & -1689.8185 & -307.2240 & -1997.0581 & -40.9 \\
0.62 & -1689.8179 & -307.2240 & -1997.0613 & -50.9 \\
0.64 & -1689.8176 & -307.2240 & -1997.0629 & -55.7 \\
0.66 & -1689.8173 & -307.2240 & -1997.0630 & -56.8 \\
0.68 & -1689.8171 & -307.2240 & -1997.0616 & -53.6 \\
0.70 & -1689.8169 & -307.2240 & -1997.0599 & -49.7 \\
0.75 & -1689.8165 & -307.2240 & -1997.0545 & -36.4 \\
0.80 & -1689.8163 & -307.2240 & -1997.0535 & -34.5 \\
1.0 & -1689.8159 & -307.2240 & -1997.0524 & -32.7 \\
2.0 & -1689.8157 & -307.2240 & -1997.0517 & -31.3 \\
single sheet & -844.9078 & -307.2240 & -1152.1438 & -31.2 \\
\hline
\end{tabular}


Table S11. Binding energies $\left(\boldsymbol{E}_{\mathbf{b d}}\right)$ of phenol adsorption between dual parallel PBH sheets $(\mathrm{Ba} 400, \mathbf{D})$ at an inter-sheet distance of $0.68 \mathrm{~nm}$

\begin{tabular}{ccccc}
\hline & Sheet $(\mathrm{Ha})$ & Phenol $(\mathrm{Ha})$ & Sheet-Phenol (Ha) & $\boldsymbol{E}_{\mathbf{b d}}\left(\mathrm{kJ} \mathrm{mol}^{-1}\right)$ \\
\hline Ba200 & -771.0762 & -307.2240 & -1078.3109 & -28.0 \\
Ba250 & -1078.0855 & -307.2240 & -1385.3230 & -35.4 \\
Ba300 & -1385.1228 & -307.2240 & -1692.3634 & -43.6 \\
Ba400 & -1689.8171 & -307.2240 & -1997.0616 & -53.6 \\
Ba550 & -2301.5132 & -307.2240 & -2608.7632 & -68.4 \\
Ba700 & -4134.3576 & -307.2240 & -4441.6117 & -78.9 \\
\hline
\end{tabular}

Table S12. Binding energies $\left(\boldsymbol{E}_{\mathbf{b d}}\right)$ of two parallel-planar molecules adsorbed in a bilayer between parallel PBH sheets placed $1 \mathrm{~nm}$ apart.

\begin{tabular}{ccccc}
\hline & Sheet $(\mathrm{Ha})$ & Compound (Ha) & Sheet-Compound (Ha) & $\boldsymbol{E}_{\mathbf{b d}}\left(\mathrm{kJ} \mathrm{mol}^{-1}\right)$ per each phenol \\
\hline & & & Phenol & \\
Ba400 & -1689.8155 & -614.4480 & -2304.2903 & -35.2 \\
Ba700 & -4134.3532 & -614.4480 & -4748.8386 & -49.1 \\
& & & Naphthalene & $-50.5^{\mathrm{a}}$ \\
Ba400 & -1689.8171 & -771.0758 & -2460.9314 & \\
\hline
\end{tabular}

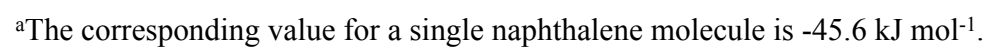



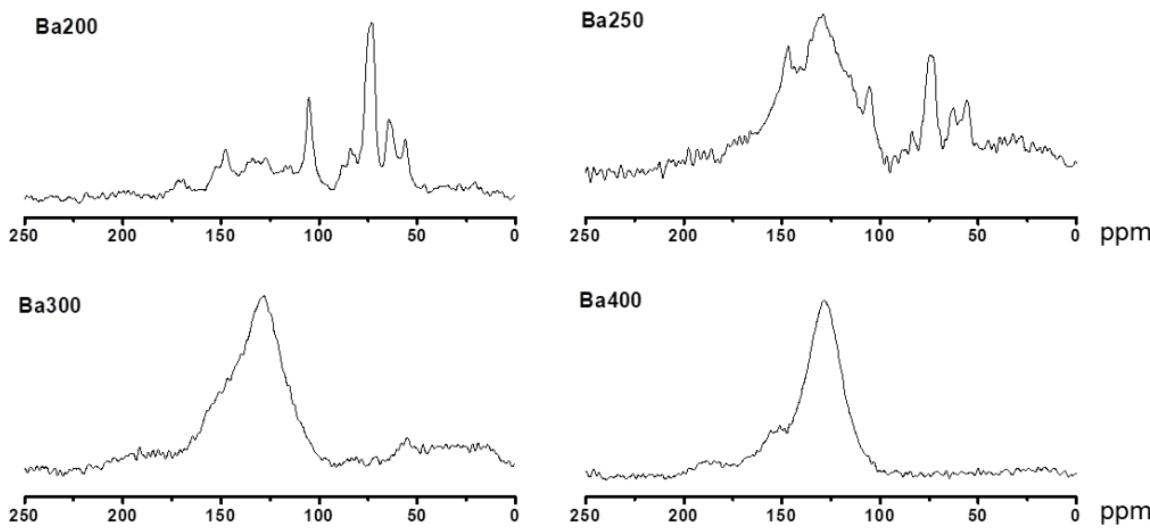

$\mathrm{Ba} 400$
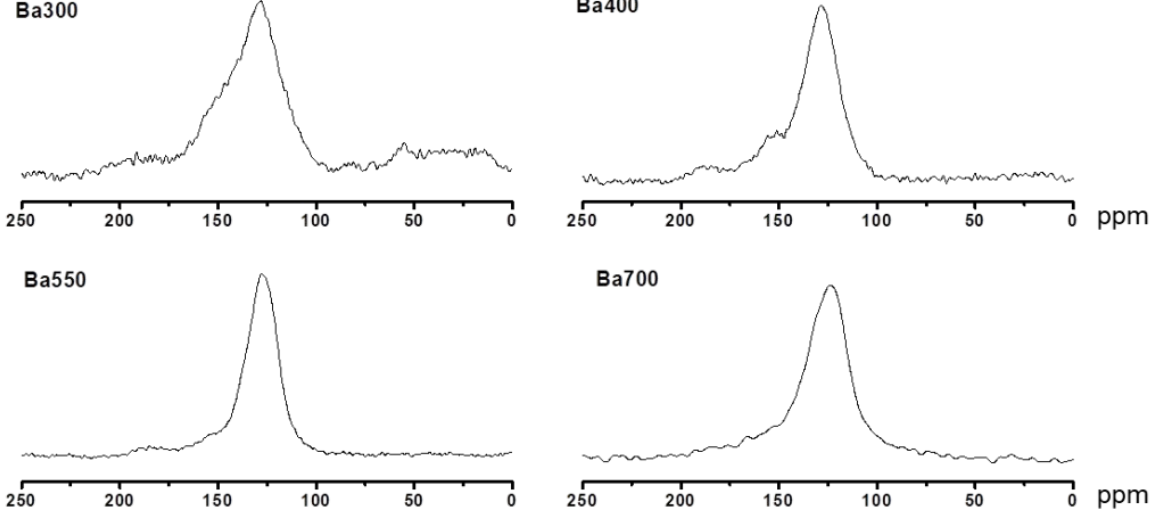

Figure S1. Solid state ${ }^{13} \mathrm{C}$ DP/MAS NMR spectra of chars

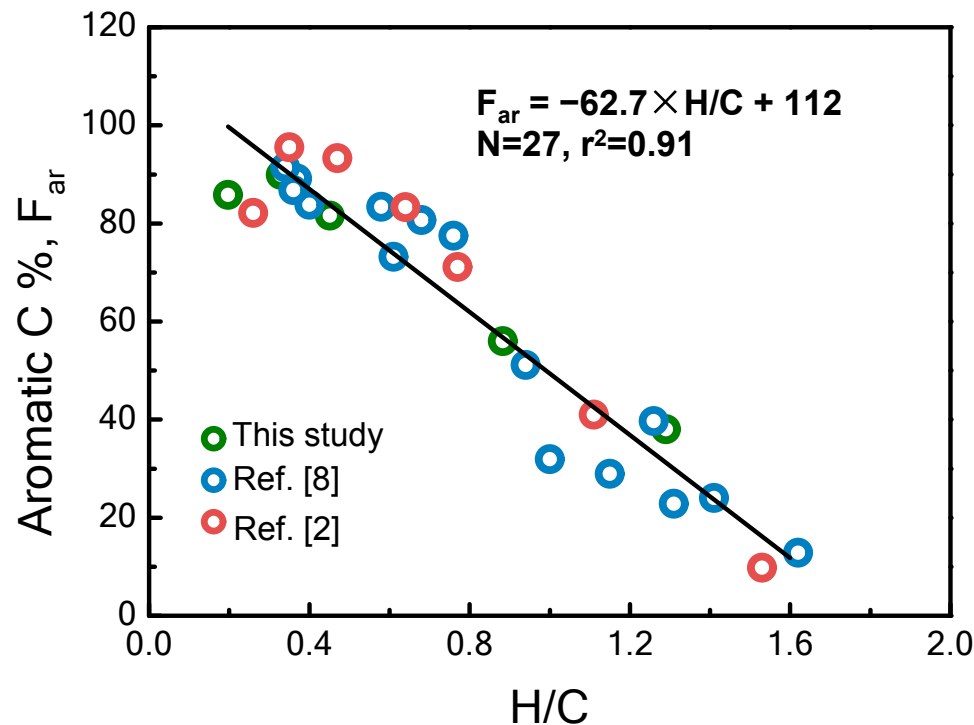

Figure S2. Correlation between the total aromatic carbon content $\left(\boldsymbol{F}_{\text {ar }}\right)$ and atomic ratio of $\mathrm{H} / \mathrm{C}$ for chars. Blue $\mathrm{e}^{8}$ and red points ${ }^{2}$ are data from literatures. Black line is the linear fitting line. 

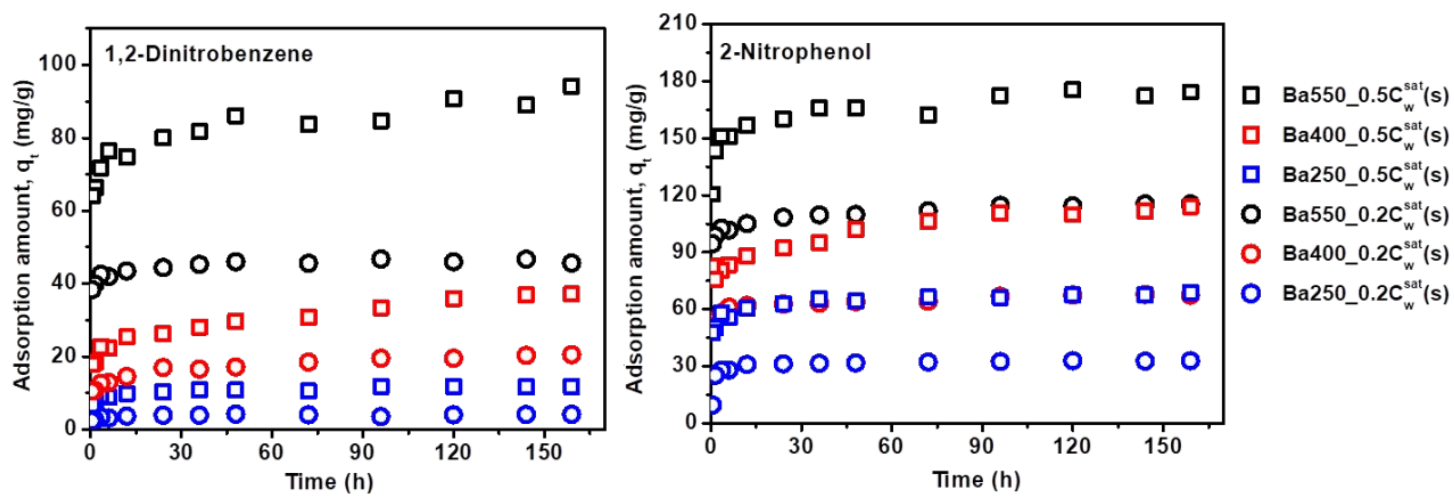

Figure S3. Preliminary kinetic experiments for two compounds with different solubilities at two initial concentrations on $\mathrm{Ba} 550, \mathrm{Ba} 400$ and $\mathrm{Ba} 250$ which represents chars produced at high HTT, medium HTT and low HTT, respectively. 

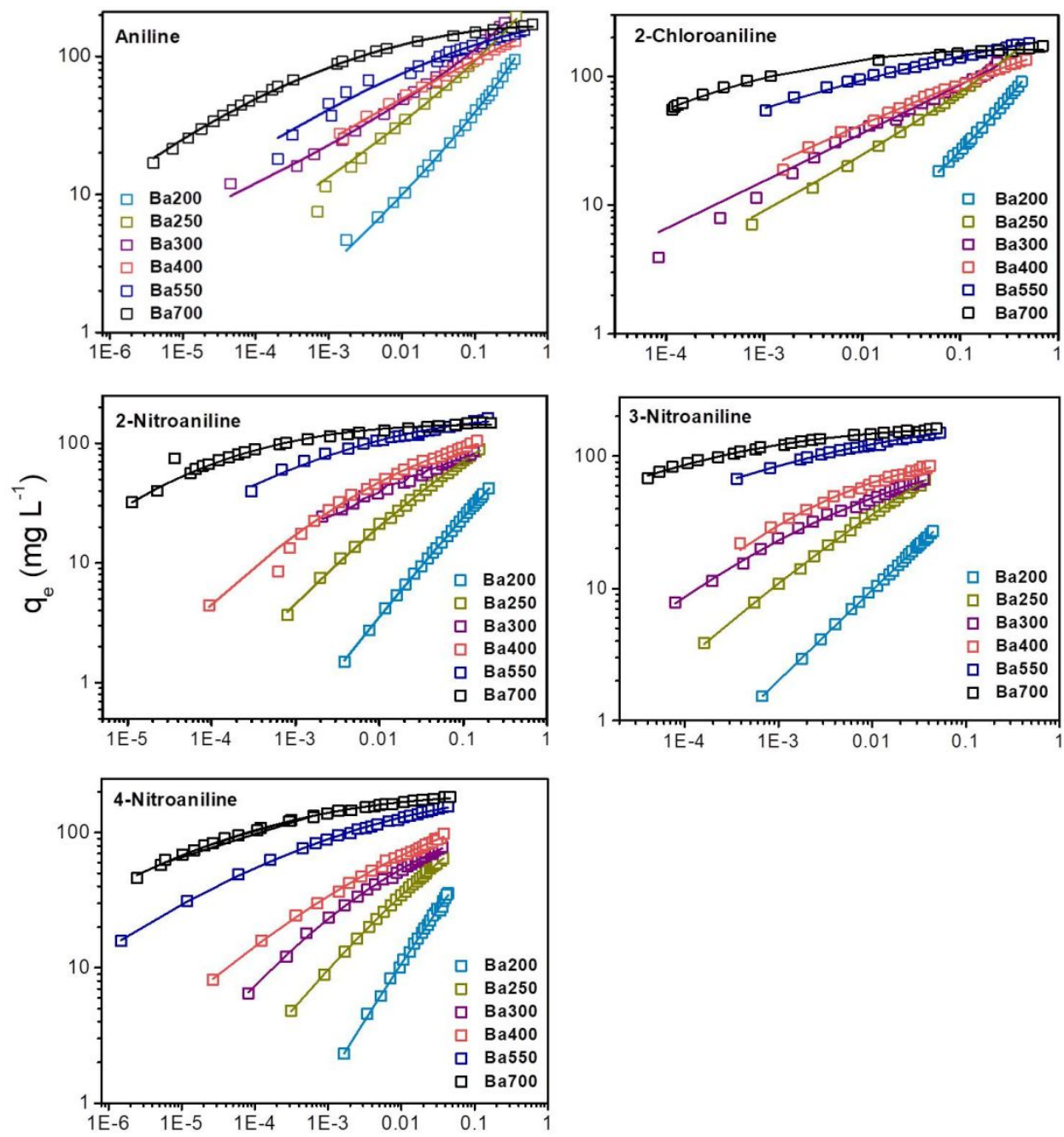

Equilibrium concentration, $C_{e} / C_{w}^{\text {sat }}(L)$

Figure S4. Adsorption isotherms of anilines on chars fit to the Dubinin-Ashtakhov model. 

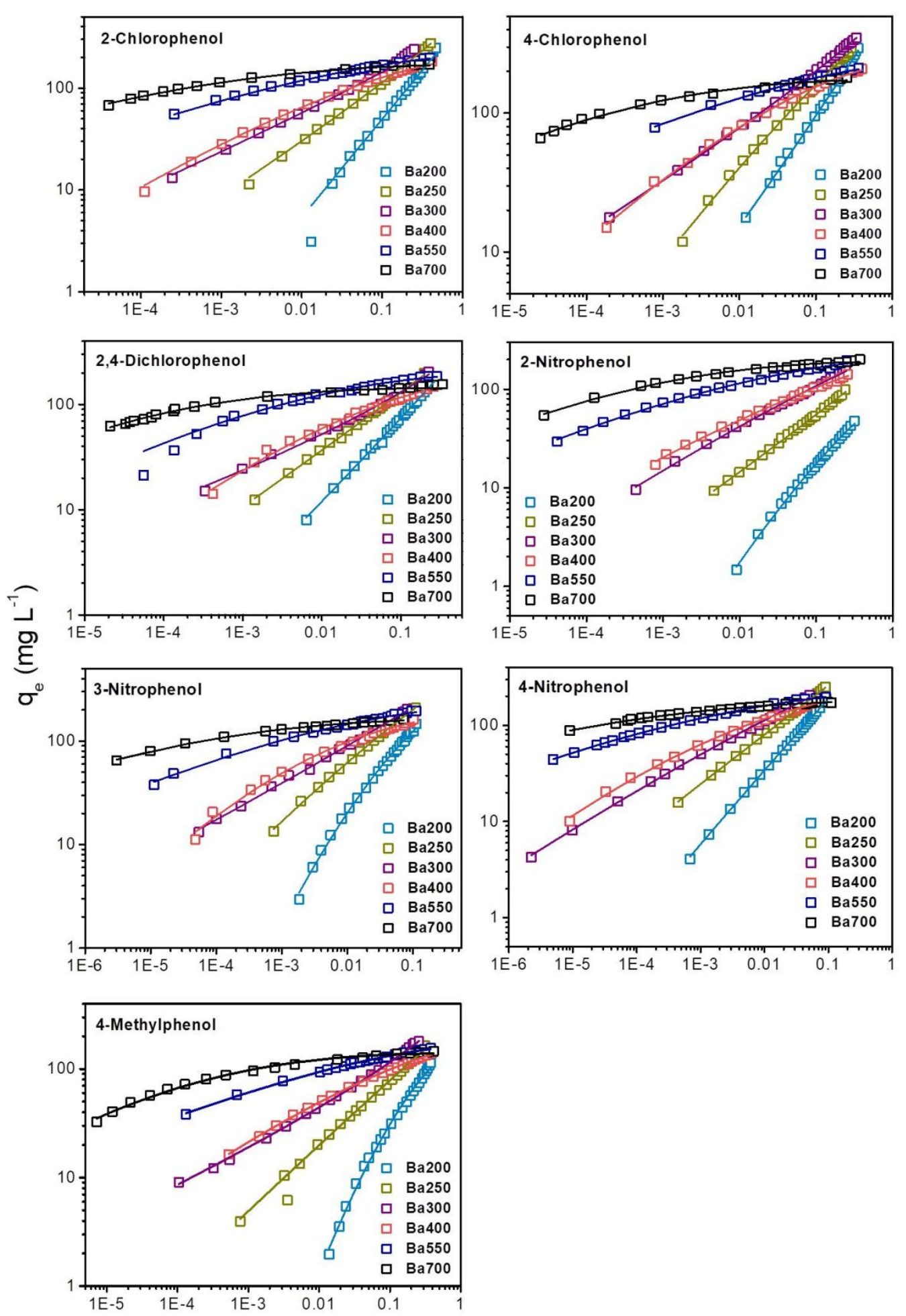

Equilibrium concentration, $\mathrm{C}_{\mathrm{e}} / \mathrm{C}_{\mathrm{w}}^{\text {sat }}(\mathrm{L})$

Figure S5. Adsorption isotherms of phenols on chars fit to the Dubinin-Ashtakhov model. 

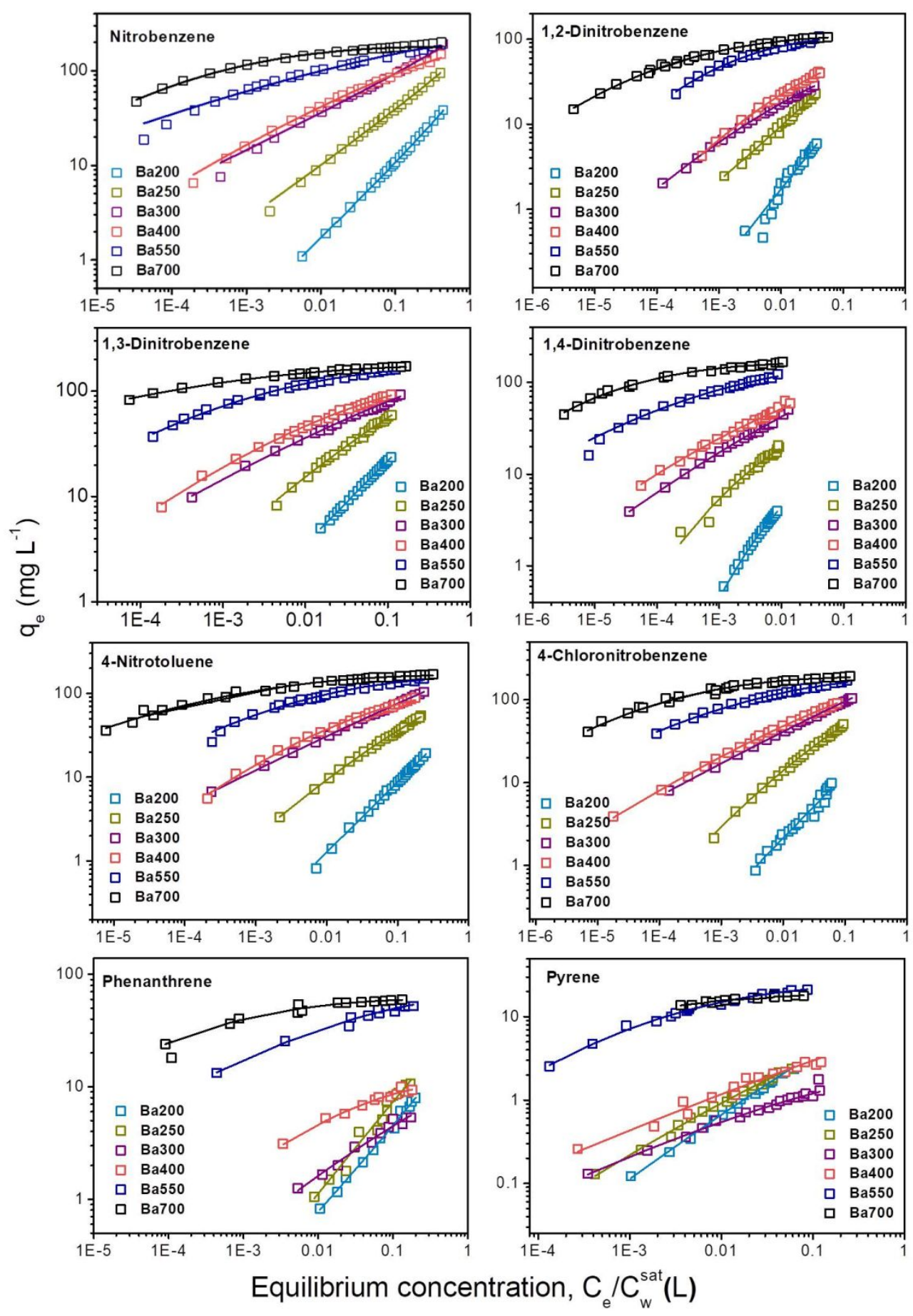

Figure S6. Adsorption isotherms of nitrobenzenes and PAHs on chars fit to the Dubinin-Ashtakhov model. 

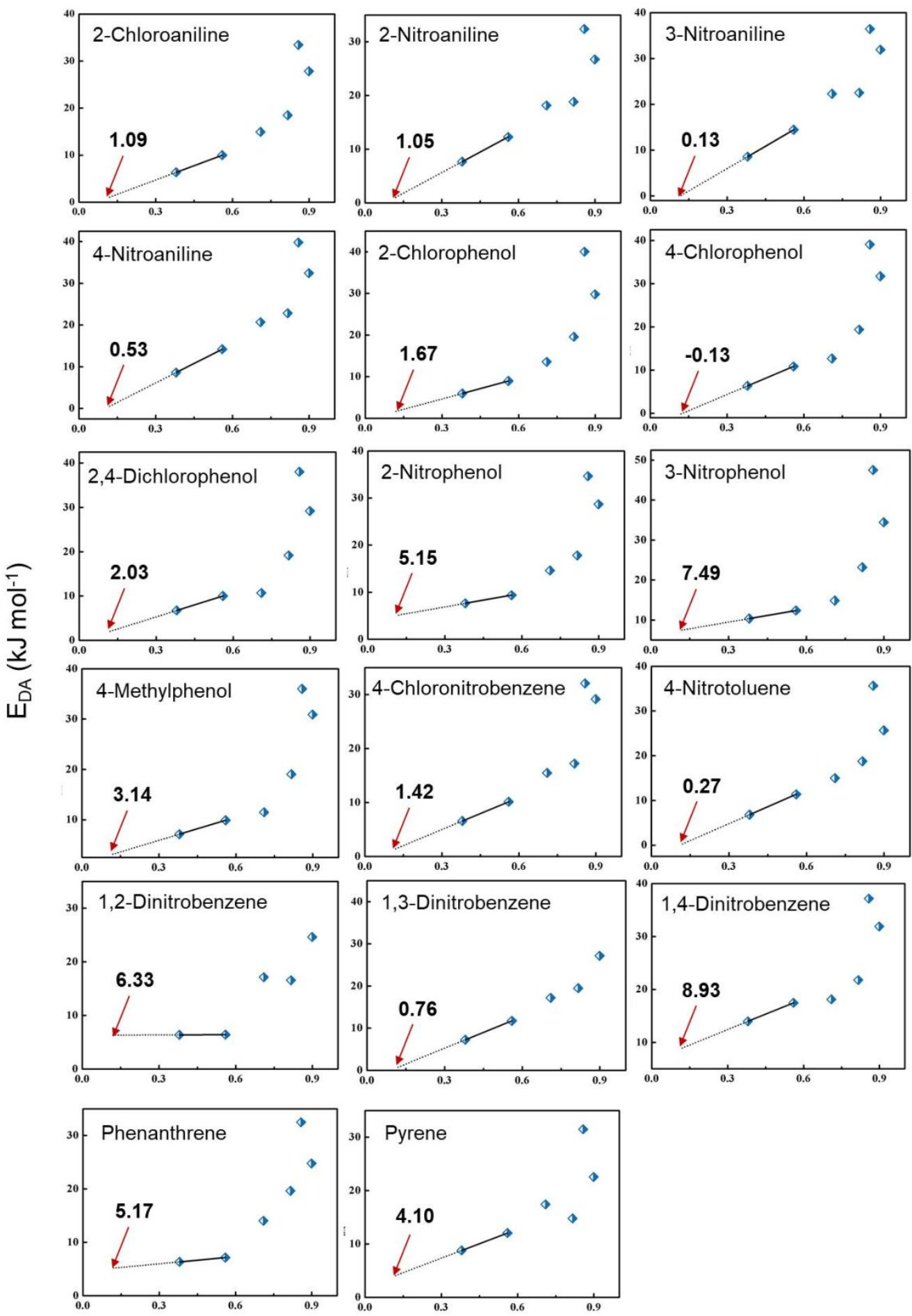

Fraction of aromatic carbon, $\mathrm{F}_{\mathrm{ar}}$

Figure S7. Correlations between the fraction of aromatic carbon $\left(\boldsymbol{F}_{\mathbf{a r}}\right)$ and $\boldsymbol{E}_{\mathbf{D A}}$ values.

Solid lines connect Ba200 and Ba250 and dotted lines are the extraploted line to the $\boldsymbol{F}_{\text {ar }}$ corresponding to fresh bamboo (0.12, red arrows). 

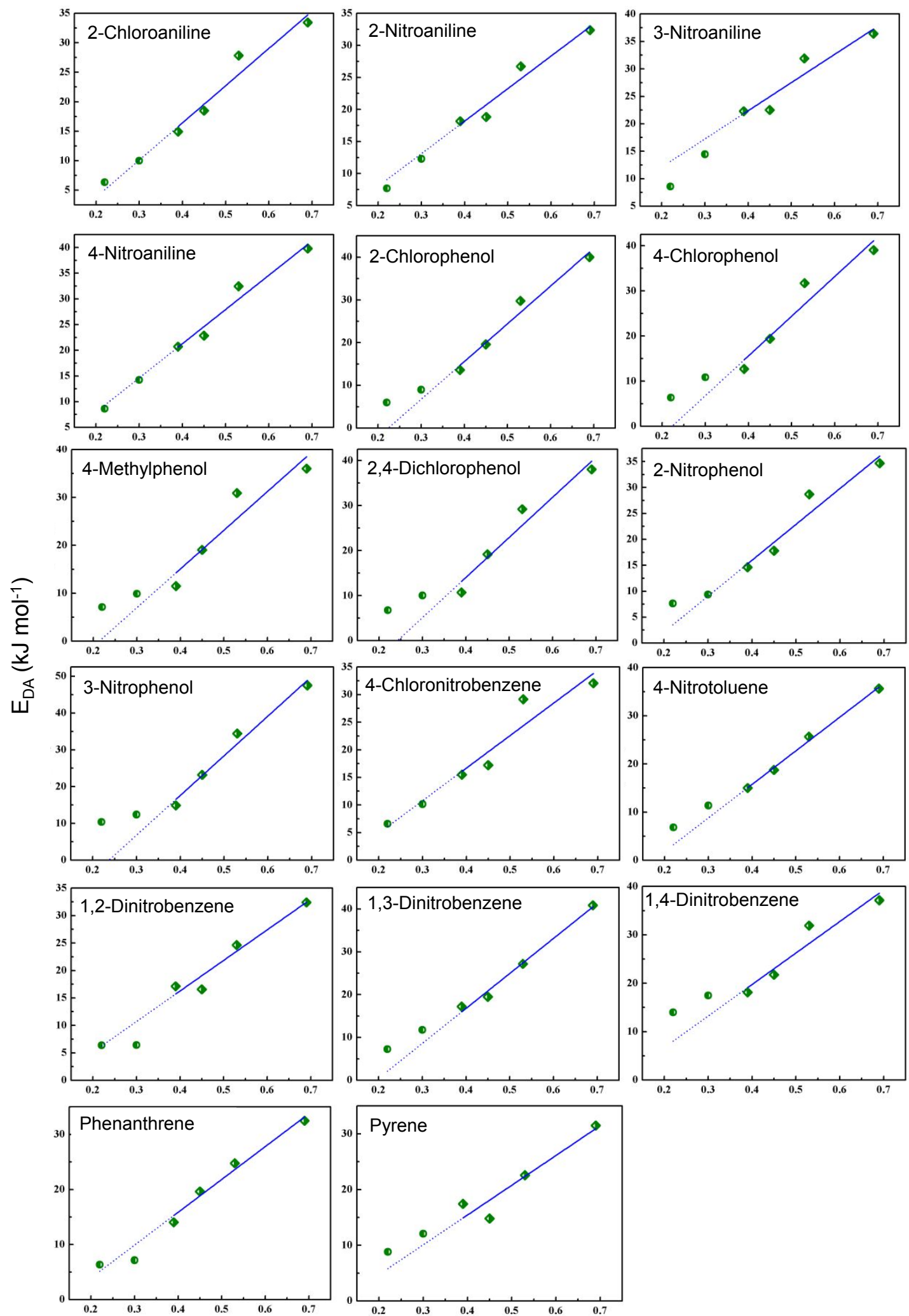

Fraction of bridgehead aromatic carbon, $\chi_{\mathrm{b}}$

Figure S8. Correlations between fraction of bridgehead aromatic carbon and $\boldsymbol{E}_{\mathbf{D A}}$

values. Solid lines are linear regression fits of Ba300, Ba400, Ba550 and Ba700. Dash lines are extrapolated lines from the solid lines. 

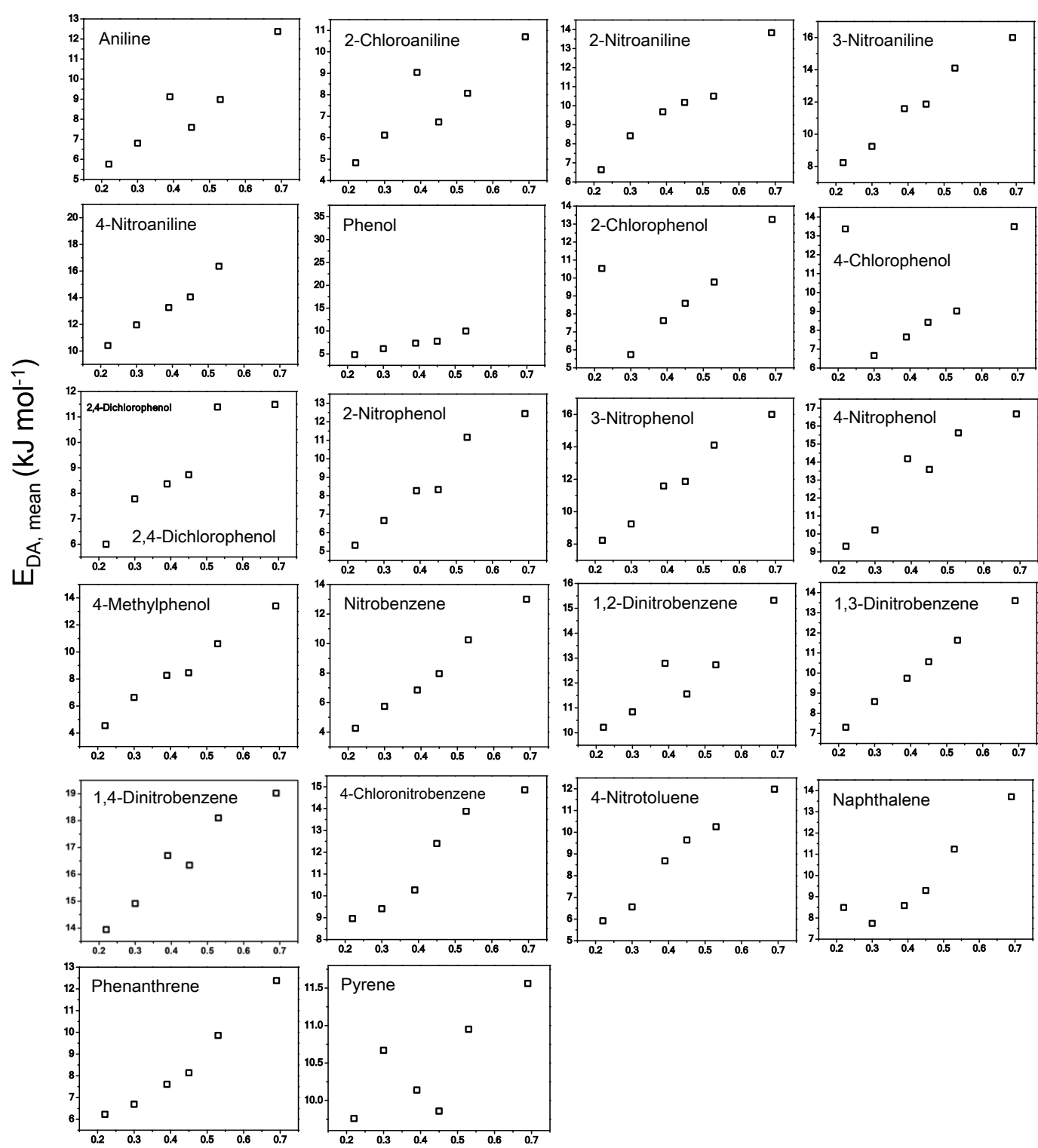

Fraction of bridgehead aromatic carbon, $\chi_{b}$

Figure S9. Correlations between fraction of bridgehead aromatic carbon $\left(\chi_{\mathbf{b}}\right)$ and $\boldsymbol{E}_{\mathbf{D A}, \text { mean }}$ values listed in Table S5. 


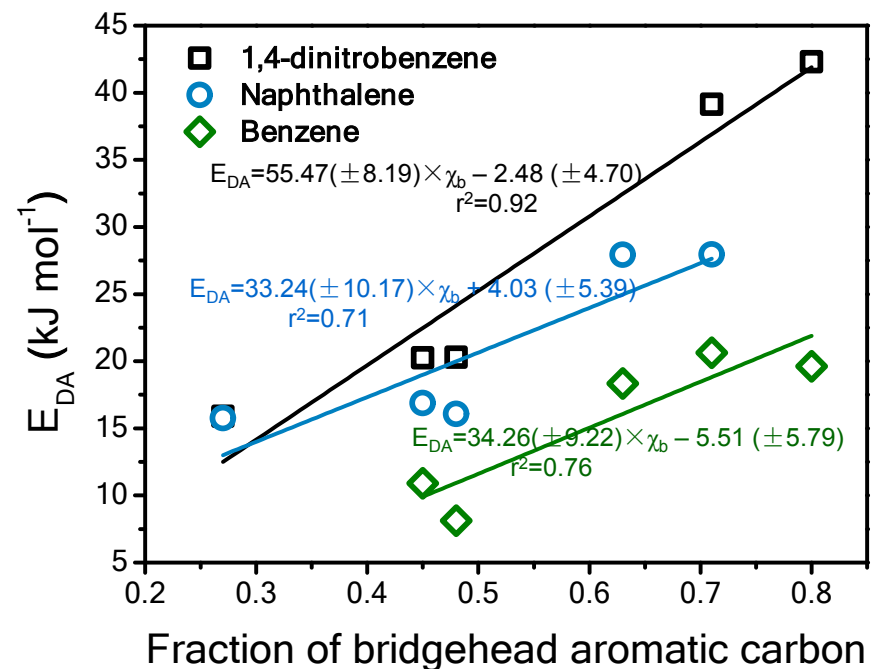

Figure S10. Correlations between sorption $\boldsymbol{E}_{\mathbf{D A}}$ values and fraction of bridgehead aromatic carbon $\left(\chi_{\mathbf{b}}\right)$ on wood chars. Data is obtained from the literature ${ }^{1,2}$. 


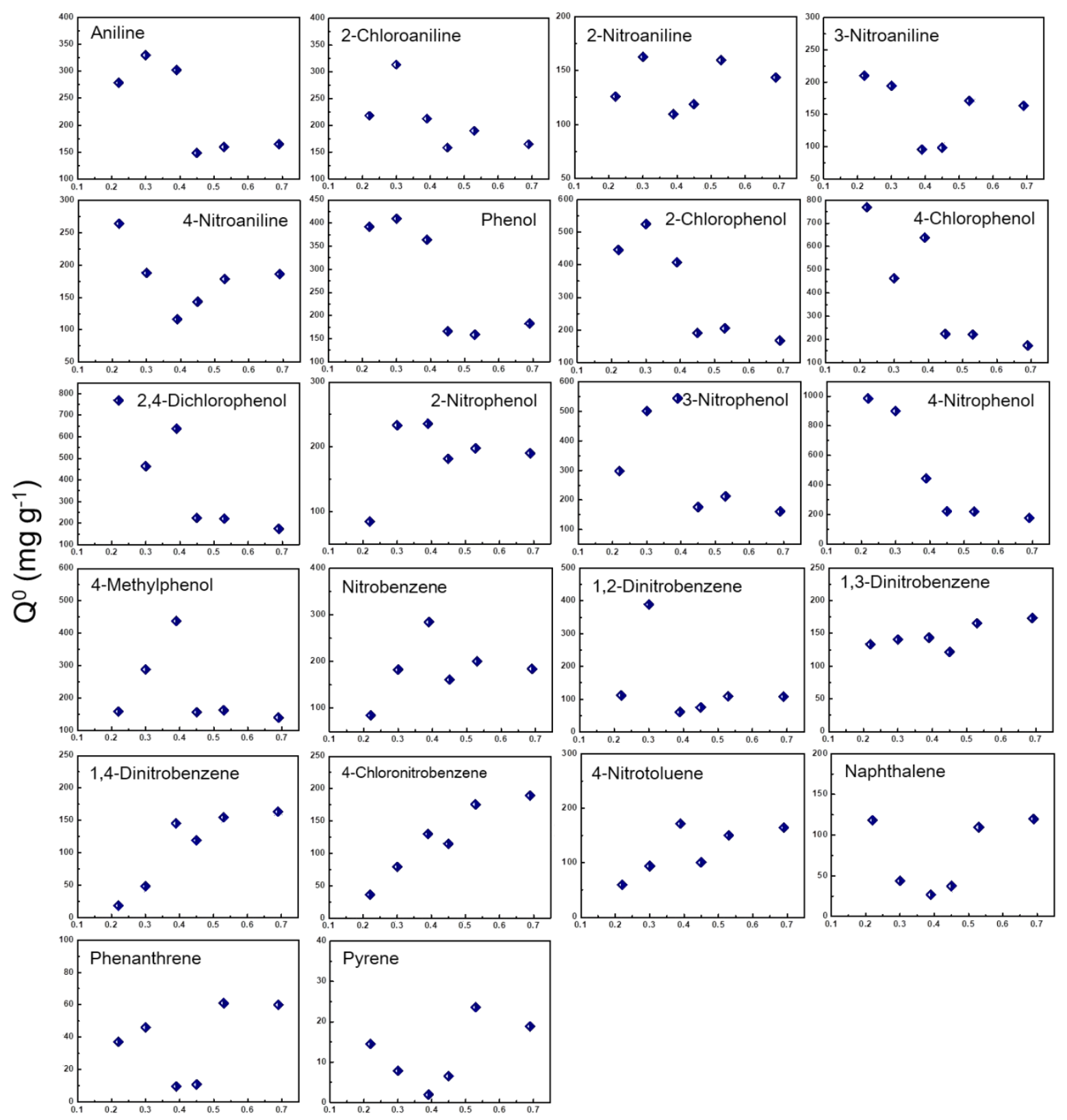

Fraction of bridgehead aromatic carbon, $\mathrm{X}_{\mathrm{b}}$

Figure S11. Correlations between sorption capacity $\left(Q^{\mathbf{0}}, \mathrm{mg} \mathrm{g}^{-1}\right)$ and fraction of bridgehead aromatic carbon $\left(\chi_{\mathbf{b}}\right)$. 
(a)

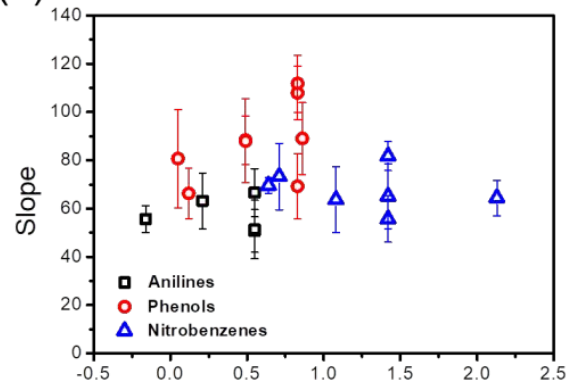

(c)

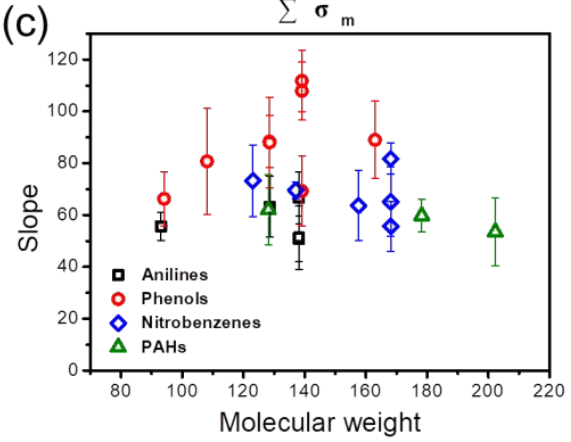

(b)

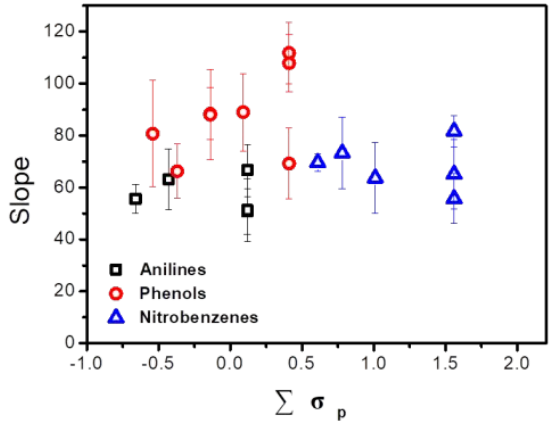

Figure S12. Correlations between the slope $m_{\mathrm{DA}}$ of the relationship $\boldsymbol{E}_{\mathbf{D A}}=m_{\mathrm{DA}} \chi_{\mathbf{b}}+$ const $_{\text {DA }}$ (from Table S4) and, (a) the sum of Hammett $\sigma_{\text {meta }}$, (b) the sum of Hammett $\sigma_{\text {para }}$, or (c) the molecular weight, for 22 compounds. 
(a) Phenol

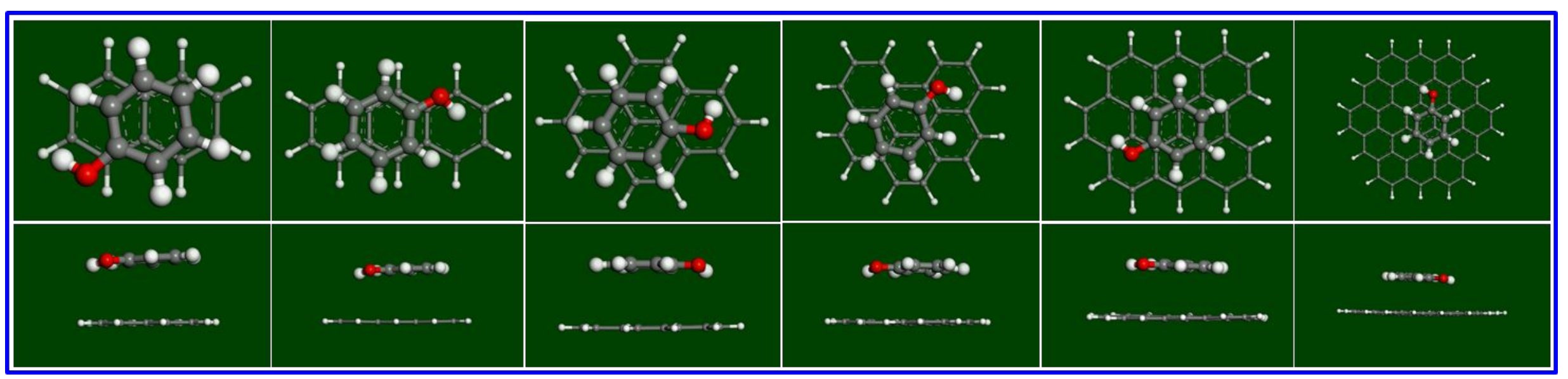

(b) Aniline

\begin{tabular}{|l|l|l|l|}
\hline \\
\hline$\infty$
\end{tabular}


(c) Nitrobenzene

(n)

(d) Naphthalene

(1)


(e) 4-Nitrophenol

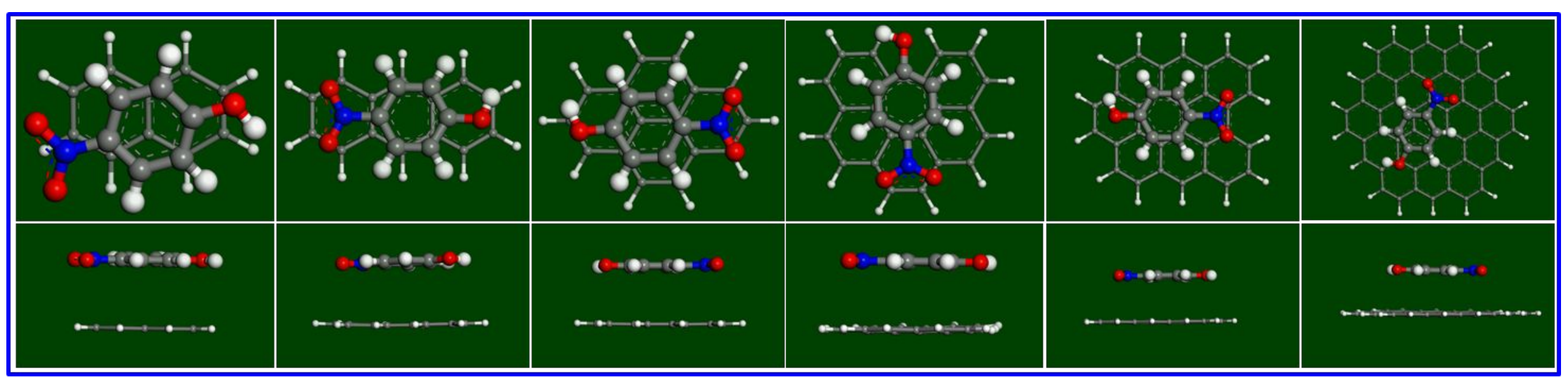

Figure S13. Simulation results for the optimized complexes. (dark gray: C; white: H; blue: N; red: O). The PBH sheets correspond, left to right, to the chars: B200, Ba250, Ba300, Ba400, Ba550, and Ba700 (structures A-F, Fig. 4a). 


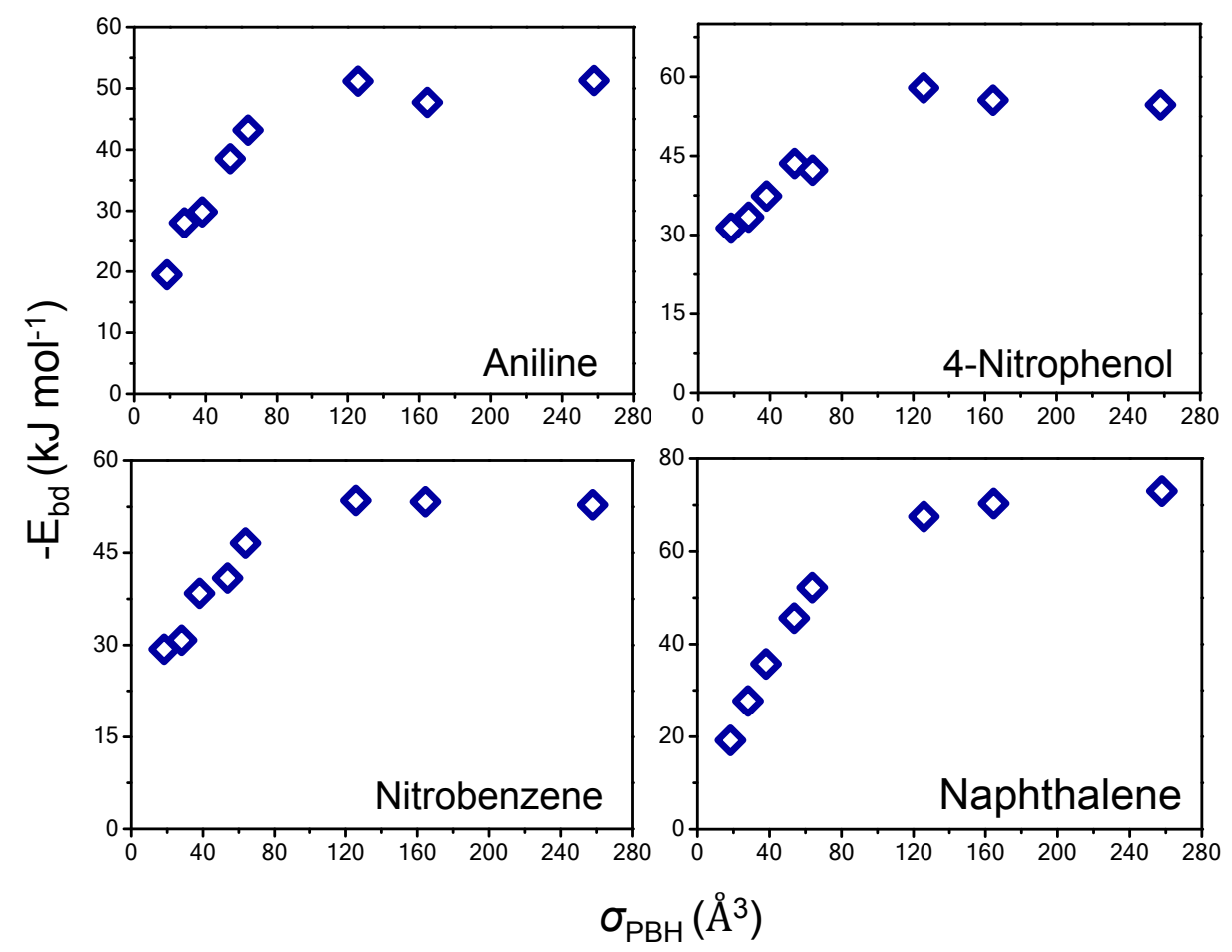

Figure S14. Correlations between static polarizability of PBH A-H (Fig. 4a) and $\boldsymbol{E}_{\mathbf{b d}}$ for adsorption of four compounds on the single-sheet PBH. 

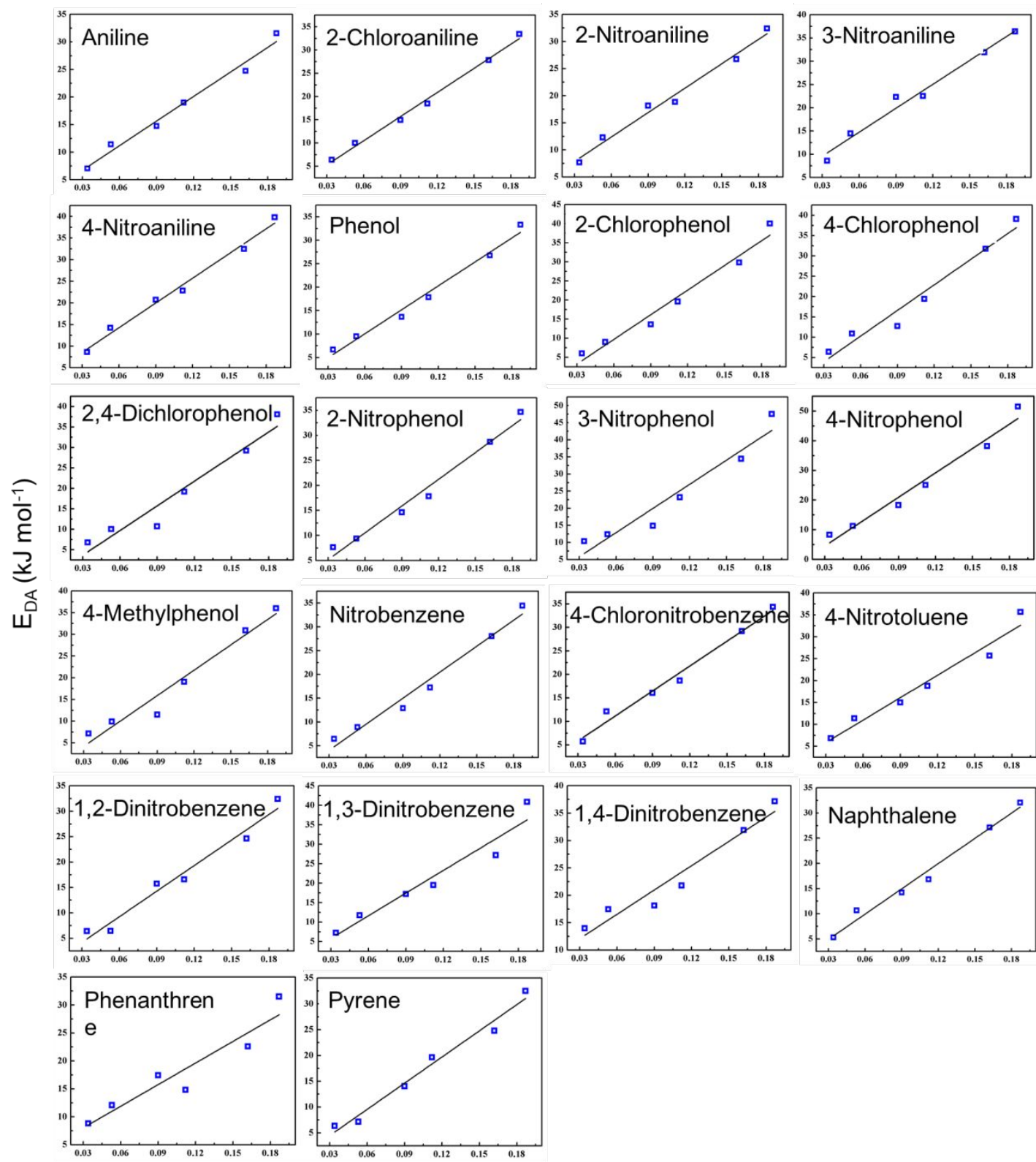

Micropore volume $\left(\mathrm{cm}^{3} \mathrm{~g}^{-1}\right)$

Figure S15. Correlations between micropore volume and $\boldsymbol{E}_{\mathbf{D A}}$ values. Solid lines are linear fits. 

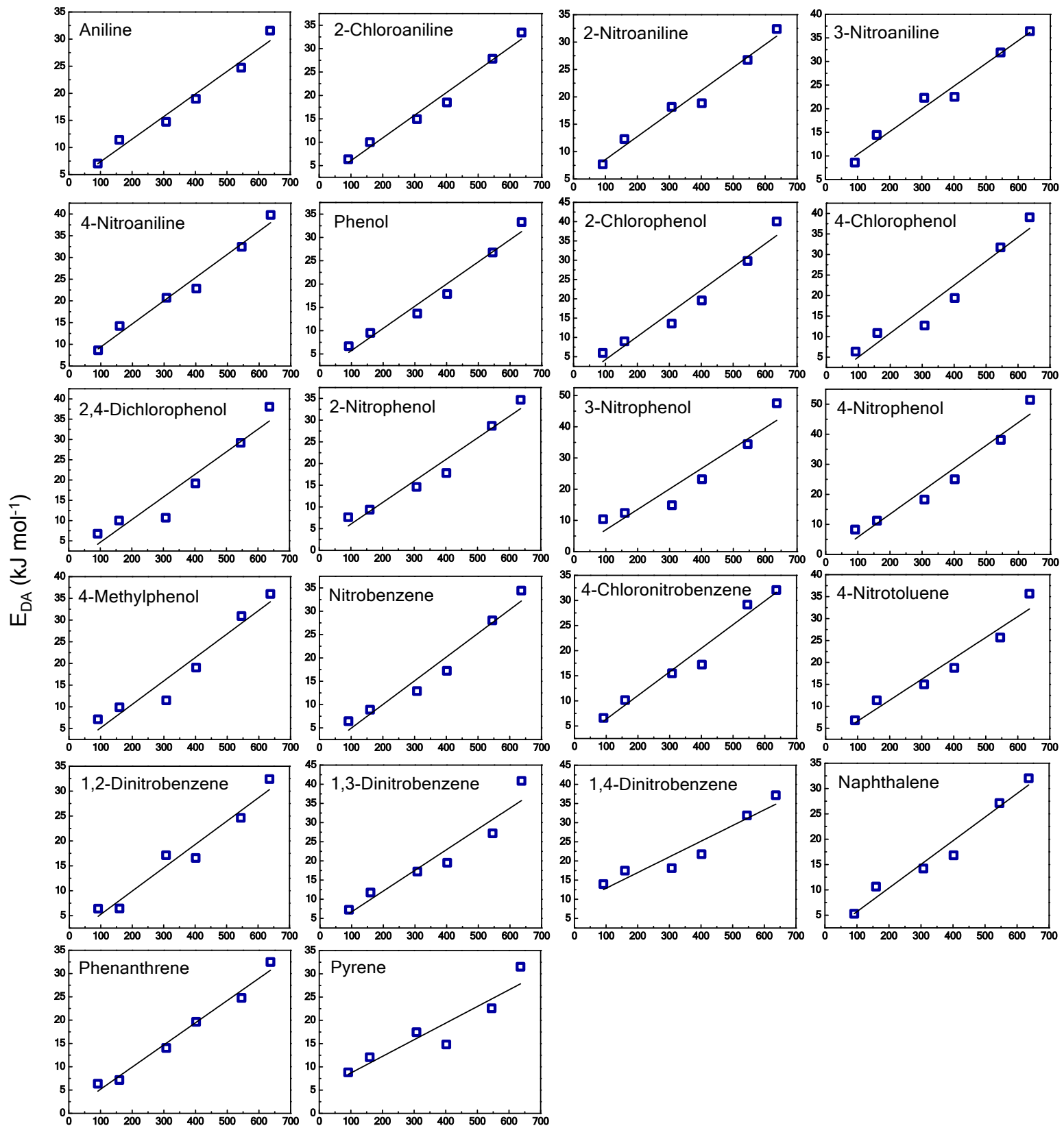

$\mathrm{CO}_{2}-\mathrm{SSA}\left(\mathrm{m}^{2} \mathrm{~g}^{-1}\right)$

Figure S16. Correlations between $\mathrm{CO}_{2}-\mathrm{SSA}$ and $\boldsymbol{E}_{\mathbf{D A}}$ values. Solid lines are linear

fits. 


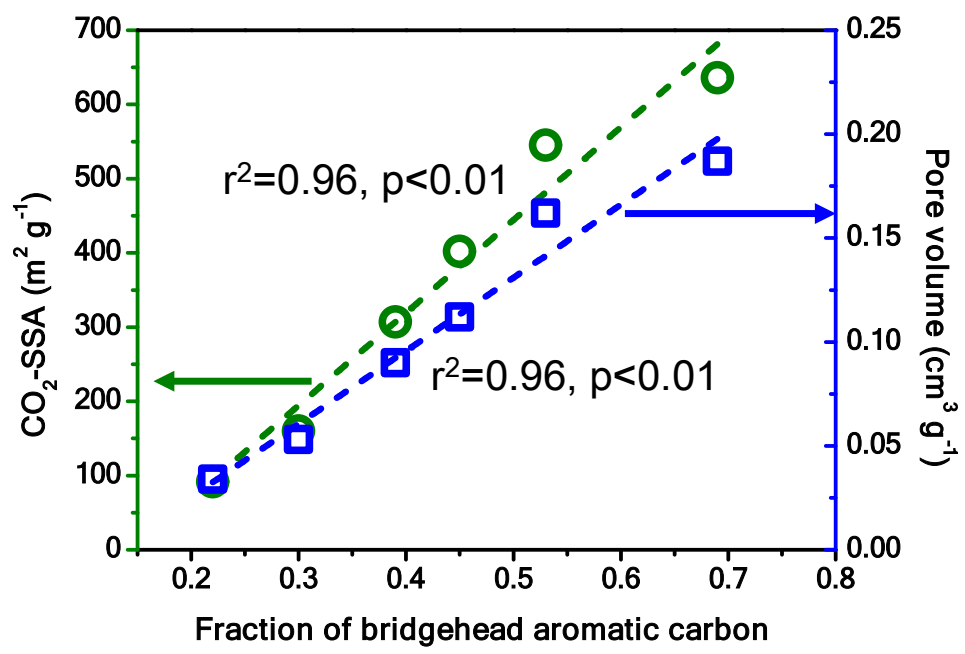

Figure S17. Correlations between fraction of bridgehead aromatic carbon and surface area and micropore volume. Short dot lines are linear fits. 


\section{Reference}

1. Lattao, C.; Cao, X.; Mao, J.; Schmidt-Rohr, K.; Pignatello, J. J., Influence of Molecular Structure and Adsorbent Properties on Sorption of Organic Compounds to a Temperature Series of Wood Chars. Environmental Science \& Technology 2014, 48, (9), 4790-4798.

2. Cao, X.; Pignatello, J. J.; Li, Y.; Lattao, C.; Chappell, M. A.; Chen, N.; Miller, L. F.; Mao, J., Characterization of Wood Chars Produced at Different Temperatures Using Advanced Solid-State C-13 NMR Spectroscopic Techniques. Energy \& Fuels 2012, 26, (9), 5983-5991.

3. Yang, K.; Yang, J.; Jiang, Y.; Wu, W.; Lin, D., Correlations and adsorption mechanisms of aromatic compounds on a high heat temperature treated bamboo biochar. Environmental Pollution 2016, 210, 57-64.

4. Willam E, A. J., Thermodynamic properties of organic compounds; enthalpy of fusion and melting point temperature compilation. Thermochimica Acta 1991, 189, $37-56$.

5. Braida, W. J.; Pignatello, J. J.; Lu, Y. F.; Ravikovitch, P. I.; Neimark, A. V.; Xing, B. S., Sorption hysteresis of benzene in charcoal particles. Environmental Science \& Technology 2003, 37, (2), 409-417.

6. Xiao, F.; Pignatello, J. J., Effects of Post-Pyrolysis Air Oxidation of Biomass Chars on Adsorption of Neutral and Ionizable Compounds. Environmental Science \& Technology 2016, 50, (12), 6276-6283.

7. Shen, X.; Guo, X.; Zhang, M.; Tao, S.; Wang, X., Sorption Mechanisms of Organic Compounds by Carbonaceous Materials: Site Energy Distribution Consideration. Environmental Science \& Technology 2015, 49, (8), 4894-4902.

8. Jin, J.; Sun, K.; Wang, Z.; Yang, Y.; Han, L.; Xing, B., Characterization and Phenanthrene Sorption of Natural and Pyrogenic Organic Matter Fractions. Environmental Science \& Technology 2017, 51, (5), 2635-2642. 